\title{
Random orthogonal matrices and the Cayley transform
}

\author{
Michael Jauch, Peter D. Hoff, and David B. Dunson \\ Department of Statistical Science \\ Duke University
}

\begin{abstract}
Random orthogonal matrices play an important role in probability and statistics, arising in multivariate analysis, directional statistics, and models of physical systems, among other areas. Calculations involving random orthogonal matrices are complicated by their constrained support. Accordingly, we parametrize the Stiefel and Grassmann manifolds, represented as subsets of orthogonal matrices, in terms of Euclidean parameters using the Cayley transform. We derive the necessary Jacobian terms for change of variables formulas. Given a density defined on the Stiefel or Grassmann manifold, these allow us to specify the corresponding density for the Euclidean parameters, and vice versa. As an application, we describe and illustrate through examples a Markov chain Monte Carlo approach to simulating from distributions on the Stiefel and Grassmann manifolds. Finally, we establish an asymptotic independent normal approximation for the distribution of the Euclidean parameters which corresponds to the uniform distribution on the Stiefel manifold. This result contributes to the growing literature on normal approximations to the entries of random orthogonal matrices or transformations thereof.
\end{abstract}

Keywords: Stiefel manifold, Grassmann manifold, Gaussian approximation, Markov chain Monte Carlo, Jacobian.

\section{Introduction}

Random orthogonal matrices play an important role in probability and statistics. They arise, for example, in multivariate analysis, directional statistics, and models of physical systems. The set of $p \times k$ orthogonal matrices $\mathcal{V}(k, p)=\left\{\boldsymbol{Q} \in \mathbb{R}^{p \times k}\right.$ $\left.\boldsymbol{Q}^{T} \boldsymbol{Q}=\boldsymbol{I}_{k}\right\}$, known as the Stiefel manifold, is a $d_{\mathcal{V}}=p k-k(k+1) / 2$ dimensional submanifold of $\mathbb{R}^{p k}$. There are two notable special cases: $\mathcal{V}(1, p)$ is equivalent to the unit hypersphere, while $\mathcal{V}(p, p)$ is equivalent to the orthogonal group $\mathcal{O}(p)$. 
Closely related to the Stiefel manifold is the Grassmann manifold $\mathcal{G}(k, p)$, the set of $k$-dimensional linear subspaces of $\mathbb{R}^{p}$. The Grassmann manifold has dimension $d_{\mathcal{G}}=(p-k) k$. Typically, points in the Grassmann manifold are thought of as equivalence classes of $\mathcal{V}(k, p)$, where two orthogonal matrices belong to the same class if they share the same column space or, equivalently, if one matrix can be obtained from the other through right multiplication by an element of $\mathcal{O}(k)$. In Section 3.2, we elaborate and expand upon the contributions of Shepard et al. [42] to provide another representation of the Grassmann manifold $\mathcal{G}(k, p)$ as the subset $\mathcal{V}^{+}(k, p)$ of $p \times k$ orthogonal matrices having a symmetric positive definite (SPD) top block. In this article, we focus on orthogonal matrices having fewer columns than rows.

Both the Stiefel and Grassmann manifolds can be equipped with a uniform probability measure, also know as an invariant or Haar measure. The uniform distribution $P_{\mathcal{V}(k, p)}$ on $\mathcal{V}(k, p)$ is characterized by its invariance to left and right multiplication by orthogonal matrices: If $\boldsymbol{Q} \sim P_{\mathcal{V}(k, p)}$, then $\boldsymbol{U} \boldsymbol{Q} \boldsymbol{V} \stackrel{\text { dist. }}{=} \boldsymbol{Q}$ for all $\boldsymbol{U} \in \mathcal{O}(p)$ and $\boldsymbol{V} \in \mathcal{O}(k)$. Letting $l: \mathcal{V}(k, p) \rightarrow \mathcal{G}(k, p)$ be the function taking an orthogonal matrix to its column space, the uniform distribution $P_{\mathcal{G}(k, p)}$ on $\mathcal{G}(k, p)$ is the pushforward measure of $P_{\mathcal{V}(k, p)}$ under $l$. In other words, the measure of $A \subseteq$ $\mathcal{G}(k, p)$ is $P_{\mathcal{G}(k, p)}[A]=P_{\mathcal{V}(k, p)}\left[l^{-1}(A)\right]$. The uniform distributions on these manifolds have a long history in probability, as we discuss in Section 6, and in statistics, where they appear in foundational work on multivariate theory [22].

Non-uniform distributions on the Stiefel and Grassmann manifolds play an important role in modern statistical applications. They are used to model directions, axes, planes, rotations, and other data lying on compact Riemannian manifolds in the field of directional statistics [31]. Also, statistical models having the Stiefel or Grassmann manifold as their parameter space are increasingly common [18, 19, 8]. In particular, Bayesian analyses of multivariate data often involve prior and posterior distributions on $\mathcal{V}(k, p)$ or $\mathcal{G}(k, p)$. Bayesian inference typically requires simulating from these posterior distributions, motivating the development of new Markov chain Monte Carlo (MCMC) methodology [19, 3, 40].

The challenge of performing calculations with random orthogonal matrices has motivated researchers to parametrize sets of square orthogonal matrices in terms of Euclidean parameters. We provide a few examples. In what Diaconis and Forrester [11] identify as the earliest substantial mathematical contribution to modern random matrix theory, Hurwitz [21] parametrizes the special orthogonal and unitary 
groups using Euler angles and computes the volumes of their invariant measures. An implication of these computations is that the Euler angles of a uniformly distributed matrix follow independent beta distributions. Anderson et al. [1] discuss the potential of various parametrizations in the simulation of uniformly distributed square orthogonal matrices. Other authors have made use of parametrizations of square orthogonal or rotation matrices in statistical applications [27, 9].

In contrast, the topic of parametrizing random orthogonal matrices having fewer columns than rows has received little attention. The recent work of Shepard et al. [42] extends four existing approaches to parametrizing square orthogonal matrices to the case when $k<p$ and to the scenario in which only the column space of the orthogonal matrix is of interest. Naturally, this latter scenario is closely related to the Grassmann manifold. The tools needed to use these parametrizations in a probabilistic setting are still largely missing.

In this article, we lay foundations for application of the Cayley parametrization of the Stiefel and Grassmann manifolds in a probabilistic setting. There are three main contributions. First, we elaborate and expand upon the work of Shepard et al. [42] to show that the Grassmann manifold $\mathcal{G}(k, p)$ can be represented by the subset $\mathcal{V}^{+}(k, p)$ of orthogonal matrices having an SPD top block and that this subset can be parametrized in terms of Euclidean elements using the Cayley transform. Next, we derive the necessary Jacobian terms for change of variables formulas. Given a density defined on $\mathcal{V}(k, p)$ or $\mathcal{V}^{+}(k, p)$, these allow us to specify the corresponding density for the Euclidean parameters, and vice versa. As an application, we describe and illustrate through examples an approach to MCMC simulation from distributions on these sets. Finally, we establish an asymptotic independent normal approximation for the distribution of the Euclidean parameters which corresponds to the uniform distribution on the Stiefel manifold. This result contributes to the growing literature on normal approximations to the entries of random orthogonal matrices or transformations thereof.

Code to replicate the figures in this article and to simulate from distributions on $\mathcal{V}(k, p)$ and $\mathcal{V}^{+}(k, p)$ is available at https://github.com/michaeljauch/cayley.

\section{Probability distributions on submanifolds of $\mathbb{R}^{n}$}

In this section, we introduce tools for defining and manipulating probability distributions on a $m$-dimensional submanifold $\mathcal{M}$ of $\mathbb{R}^{n}$. In particular, we discuss the 
reference measure with respect to which we define densities on $\mathcal{M}$, we make precise what it means for us to parametrize $\mathcal{M}$ in terms of Euclidean parameters, and we state a change of variables formula applicable in this setting. Our formulation of these ideas follows that of Diaconis et al. [15] somewhat closely. This general discussion will form the basis for our handling of the specific cases in which $\mathcal{M}$ is $\mathcal{V}(k, p)$ or $\mathcal{V}^{+}(k, p)$.

In order to specify probability distributions on $\mathcal{M}$ in terms of density functions, we need a reference measure on that space, analogous to Lebesgue measure $L^{m}$ on $\mathbb{R}^{m}$. As in Diaconis et al. [15] and Byrne and Girolami [3], we take the Hausdorff measure as our reference measure. Heuristically, the $m$-dimensional Hausdorff measure of $A \subset \mathbb{R}^{n}$ is the $m$-dimensional area of $A$. More formally, the $m$-dimensional Hausdorff measure $H^{m}(A)$ of $A$ is defined

$$
H^{m}(A)=\lim _{\delta \rightarrow 0} \inf _{\substack{A \subset \cup_{i} S_{i} \\ \operatorname{diam}\left(S_{i}\right)<\delta}} \sum_{i} \alpha_{m}\left(\frac{\operatorname{diam}\left(S_{i}\right)}{2}\right)^{m}
$$

where the infimum is taken over countable coverings $\left\{S_{i}\right\}_{i \in \mathbb{N}}$ of $A$ with

$$
\operatorname{diam}\left(S_{i}\right)=\sup \left\{|x-y|: x, y \in S_{i}\right\}
$$

and $\alpha_{m}=\Gamma\left(\frac{1}{2}\right)^{m} / \Gamma\left(\frac{m}{2}+1\right)$, the volume of the unit ball in $\mathbb{R}^{n}$. The $d_{\mathcal{V}}$-dimensional Hausdorff measure on $\mathcal{V}(k, p)$ coincides with $P_{\mathcal{V}(k, p)}$ up to a multiplicative constant.

Let $g: \mathcal{M} \rightarrow \mathbb{R}$ be proportional to a density with respect to the $m$-dimensional Hausdorff measure on $\mathcal{M}$. Furthermore, suppose $\mathcal{M}$ can be parametrized by a function $f$ from an open domain $\mathcal{D} \in \mathbb{R}^{m}$ to $\mathcal{I}=f(\mathcal{D}) \subseteq \mathcal{M}$ satisfying the following conditions:

1. Almost all of $\mathcal{M}$ is contained in the image $\mathcal{I}$ of $\mathcal{D}$ under $f$ so that $H^{m}(\mathcal{M} \backslash \mathcal{I})=$ 0

2. The function $f$ is injective on $\mathcal{D}$;

3. The function $f$ is continuously differentiable on $\mathcal{D}$ with the derivative matrix $D f(\phi)$ at $\phi \in \mathcal{D}$.

In this setting, we obtain a simple change of variables formula. Define the $m$ dimensional Jacobian of $f$ at $\phi$ as $J_{m} f(\phi)=\left|D f(\phi)^{T} D f(\phi)\right|^{1 / 2}$. Like the familiar Jacobian determinant, this term acts as a scaling factor in a change of variables 
formula. However, it is defined even when the derivative matrix is not square. For more details, see the discussion in Diaconis et al. [15]. The change of variables formula is given in the following theorem, which is essentially a restatement of the main result of Traynor [46]:

Theorem 2.1. For all Borel subsets $A \subset \mathcal{D}$

$$
\int_{A} J_{m} f(\phi) L^{m}(d \phi)=H^{m}[f(A)]
$$

and hence

$$
\int_{A} g[f(\boldsymbol{\phi})] J_{m} f(\boldsymbol{\phi}) L^{m}(d \boldsymbol{\phi})=\int_{f(A)} g(\boldsymbol{y}) H^{m}(d \boldsymbol{y})
$$

Naturally, the change of variables formula has an interpretation in terms of random variables. Let $\boldsymbol{y}$ be a random element of $\mathcal{M}$ whose distribution has a density proportional to $g$. Then $\boldsymbol{y} \stackrel{\text { dist. }}{=} f(\phi)$ when the distribution of $\phi \in \mathcal{D}$ has a density proportional to $g[f(\phi)] J_{m} f(\phi)$.

\section{The Cayley parametrizations}

The Cayley transform, as introduced in Cayley [5], is a map from skew-symmetric matrices to special orthogonal matrices. Given $\boldsymbol{X}$ in $\operatorname{Skew}(p)=\left\{\boldsymbol{X} \in \mathbb{R}^{p \times p} \mid \boldsymbol{X}=\right.$ $\left.-\boldsymbol{X}^{T}\right\}$, the (original) Cayley transform of $\boldsymbol{X}$ is the special orthogonal matrix

$$
C_{\text {orig. }}(\boldsymbol{X})=\left(\boldsymbol{I}_{p}+\boldsymbol{X}\right)\left(\boldsymbol{I}_{p}-\boldsymbol{X}\right)^{-1} \text {. }
$$

We work instead with a modified version of the Cayley transform described in Shepard et al. [42]. In this version, the Cayley transform of $\boldsymbol{X}$ is the $p \times k$ orthogonal matrix

$$
C(\boldsymbol{X})=\left(\boldsymbol{I}_{p}+\boldsymbol{X}\right)\left(\boldsymbol{I}_{p}-\boldsymbol{X}\right)^{-1} \boldsymbol{I}_{p \times k}
$$

where $\boldsymbol{I}_{p \times k}$ denotes the $p \times k$ matrix having the identity matrix as its top block and the remaining entries zero. The matrix $\boldsymbol{I}_{p}-\boldsymbol{X}$ is invertible for any $\boldsymbol{X} \in \operatorname{Skew}(p)$, so the Cayley transform is defined everywhere.

In this section, we parametrize (in the sense of Section 2) the sets $\mathcal{V}(k, p)$ and $\mathcal{V}^{+}(k, p)$ using the Cayley transform $C$. We are able to parametrize these distinct sets by restricting the domain of $C$ to distinct subsets of Skew $(p)$. The third condition of 
the previous section requires that $C$ be continuously differentiable on its domain. We verify this condition by computing the derivative matrices of $C$, clearing a path for the statement of change of variables formulas in Section 4 . We also state and discuss an important proposition which justifies our claim that the Grassmann manifold $\mathcal{G}(k, p)$ can be represented by the set $\mathcal{V}^{+}(k, p)$.

\subsection{Cayley parametrization of the Stiefel manifold}

To parametrize the Stiefel manifold $\mathcal{V}(k, p)$, the domain of $C$ is restricted to the subset

$$
\mathcal{D}_{\mathcal{V}}=\left\{\boldsymbol{X}=\left[\begin{array}{cc}
\boldsymbol{B} & -\boldsymbol{A}^{T} \\
\boldsymbol{A} & \mathbf{0}_{p-k}
\end{array}\right] \mid \begin{array}{l}
\boldsymbol{B} \in \mathbb{R}^{k \times k} \in \operatorname{Skew}(k) \\
\boldsymbol{A} \in \mathbb{R}^{p-k \times k}
\end{array}\right\} \subset \operatorname{Skew}(p)
$$

Let $\boldsymbol{X} \in \mathcal{D}_{\mathcal{V}}$ and set $\boldsymbol{Q}=C(\boldsymbol{X})$. Partition $\boldsymbol{Q}=\left[\begin{array}{ll}\boldsymbol{Q}_{1}^{T} & \boldsymbol{Q}_{2}^{T}\end{array}\right]^{T}$ so that $\boldsymbol{Q}_{1}$ is square. We can write the blocks of $\boldsymbol{Q}$ in terms of the blocks of $\boldsymbol{X}$ as

$$
\begin{aligned}
& \boldsymbol{Q}_{1}=\left(\boldsymbol{I}_{k}-\boldsymbol{A}^{T} \boldsymbol{A}+\boldsymbol{B}\right)\left(\boldsymbol{I}_{k}+\boldsymbol{A}^{T} \boldsymbol{A}-\boldsymbol{B}\right)^{-1} \\
& \boldsymbol{Q}_{2}=2 \boldsymbol{A}\left(\boldsymbol{I}_{k}+\boldsymbol{A}^{T} \boldsymbol{A}-\boldsymbol{B}\right)^{-1} .
\end{aligned}
$$

The matrix $\boldsymbol{I}_{k}+\boldsymbol{A}^{T} \boldsymbol{A}-\boldsymbol{B}$ is the sum of a symmetric positive definite matrix and a skew-symmetric matrix and therefore nonsingular (see the appendix for a proof). This observation guarantees (again) that the Cayley transform is defined for all $\boldsymbol{X} \in \mathcal{D}_{\mathcal{V}}$. We can recover the matrices $\boldsymbol{A}$ and $\boldsymbol{B}$ from $\boldsymbol{Q}$ :

$$
\begin{aligned}
\boldsymbol{F} & =\left(\boldsymbol{I}_{k}-\boldsymbol{Q}_{1}\right)\left(\boldsymbol{I}_{k}+\boldsymbol{Q}_{1}\right)^{-1} \\
\boldsymbol{B} & =\frac{1}{2}\left(\boldsymbol{F}^{T}-\boldsymbol{F}\right) \\
\boldsymbol{A} & =\frac{1}{2} \boldsymbol{Q}_{2}\left(\boldsymbol{I}_{k}+\boldsymbol{F}\right) .
\end{aligned}
$$

We are now in a position to verify the first two conditions of Section 2. The image of $\mathcal{D}_{V}$ under $C$ is the set

$$
\mathcal{I}_{\mathcal{V}}=\left\{\boldsymbol{Q}=\left[\begin{array}{ll}
\boldsymbol{Q}_{1}^{T} & \boldsymbol{Q}_{2}^{T}
\end{array}\right]^{T} \in \mathcal{V}(k, p) \mid \boldsymbol{I}_{k}+\boldsymbol{Q}_{1} \text { is nonsingular }\right\}
$$

which has measure one with respect to $P_{\mathcal{V}(k, p)}$. The injectivity of $C$ on $\mathcal{D}_{\mathcal{V}}$ follows from the existence of the inverse mapping described in equations $1-3$. All that 
remains to be verified is the third condition, that $C$ is continuously differentiable on its domain.

As the first step in computing the derivative matrix of $C$, we define a $d \mathcal{V}^{-}$ dimensional vector $\boldsymbol{\varphi}$ containing each of the independent entries of $\boldsymbol{X}$. Let $\boldsymbol{b}$ be the $k(k-1) / 2$-dimensional vector of independent entries of $\boldsymbol{B}$ obtained by eliminating diagonal and supradiagonal elements from the vectorization vec $\boldsymbol{B}$. The vector $\boldsymbol{\varphi}=\left(\boldsymbol{b}^{T}, \operatorname{vec} \boldsymbol{A}^{T}\right)^{T}$ then contains each of the independent entries of $\boldsymbol{X}$. Let $\boldsymbol{X}_{\boldsymbol{\varphi}} \in \mathcal{D}_{\mathcal{V}}$ be the matrix having $\varphi$ as its corresponding vector of independent entries.

The Cayley transform can now be thought of as a function of $\varphi$ :

$$
C(\boldsymbol{\varphi})=\left(\boldsymbol{I}_{p}+\boldsymbol{X}_{\boldsymbol{\varphi}}\right)\left(\boldsymbol{I}_{p}-\boldsymbol{X}_{\boldsymbol{\varphi}}\right)^{-1} \boldsymbol{I}_{p \times k}
$$

As a function of $\boldsymbol{\varphi}$, the Cayley transform is a bijection between the set $\mathcal{D}_{\mathcal{V}}^{\varphi}=\{\boldsymbol{\varphi} \in$ $\left.\mathbb{R}^{d_{\mathcal{V}}}: \boldsymbol{X}_{\varphi} \in \mathcal{D}_{\mathcal{V}}\right\}=\mathbb{R}^{d_{\mathcal{V}}}$ and $\mathcal{I}_{\mathcal{V}}$. The inverse Cayley transform is defined in the obvious way as the map $C^{-1}: \boldsymbol{Q} \mapsto\left(\boldsymbol{b}^{T} \text {, vec } \boldsymbol{A}^{T}\right)^{T}$ where $\boldsymbol{B}$ and $\boldsymbol{A}$ are computed from $\boldsymbol{Q}$ according to equations 1-3 and $\boldsymbol{b}$ contains the independent entries of $\boldsymbol{B}$ as before.

The next lemma provides an explicit linear map $\boldsymbol{\Gamma}_{\mathcal{V}}$ from $\boldsymbol{\varphi}$ to vec $\boldsymbol{X}_{\boldsymbol{\varphi}}$, greatly simplifying our calculation of the derivative matrix $D C(\boldsymbol{\varphi})$. The entries of $\boldsymbol{\Gamma}_{\mathcal{V}}$ belong to the set $\{-1,0,1\}$. The construction of $\boldsymbol{\Gamma}_{\mathcal{V}}$ involves the commutation matrix $\boldsymbol{K}_{p, p}$ and the matrix $\widetilde{\boldsymbol{D}}_{k}$ satisfying $\widetilde{\boldsymbol{D}}_{k} \boldsymbol{b}=\operatorname{vec} \boldsymbol{B}$, both of which are discussed in Magnus [28] and defined explicitly in Appendix B . Set $\boldsymbol{\Theta}_{1}=\left[\begin{array}{ll}\boldsymbol{I}_{k} & \mathbf{0}_{k \times p-k}\end{array}\right]$ and $\boldsymbol{\Theta}_{2}=$ $\left[\begin{array}{ll}\mathbf{0}_{p-k \times k} & \boldsymbol{I}_{p-k}\end{array}\right]$.

Lemma 3.0.1. The equation $\operatorname{vec} X_{\varphi}=\boldsymbol{\Gamma}_{\mathcal{V}} \boldsymbol{\varphi}$ is satisfied by the matrix

$$
\boldsymbol{\Gamma}_{\mathcal{V}}=\left[\left(\boldsymbol{\Theta}_{1}^{T} \otimes \boldsymbol{\Theta}_{1}^{T}\right) \widetilde{\boldsymbol{D}}_{k} \quad\left(\boldsymbol{I}_{p^{2}}-\boldsymbol{K}_{p, p}\right)\left(\boldsymbol{\Theta}_{1}^{T} \otimes \boldsymbol{\Theta}_{2}^{T}\right)\right]
$$

With these pieces in place, we can now identify the derivative matrix $C(\boldsymbol{\varphi})$.

Proposition 3.1. The Cayley transform is continuously differentiable on $\mathcal{D}_{\mathcal{V}}^{\varphi}=\mathbb{R}^{d_{\mathcal{V}}}$ with derivative matrix

$$
D C(\boldsymbol{\varphi})=2\left[\boldsymbol{I}_{p \times k}^{T}\left(\boldsymbol{I}_{p}-\boldsymbol{X}_{\boldsymbol{\varphi}}\right)^{-T} \otimes\left(\boldsymbol{I}_{p}-\boldsymbol{X}_{\boldsymbol{\varphi}}\right)^{-1}\right] \boldsymbol{\Gamma}_{\mathcal{V}}
$$

The form of the derivative matrix reflects the composite structure of the Cayley transform as a function of $\varphi$. The Kronecker product term arises from differentiating 
$C$ with respect to $\boldsymbol{X}_{\boldsymbol{\varphi}}$, while the matrix $\boldsymbol{\Gamma}_{\mathcal{V}}$ arises from differentiating $\boldsymbol{X}_{\boldsymbol{\varphi}}$ with respect to $\varphi$.

\subsection{The Cayley parametrization of the Grassmann manifold}

While the definition of the Grassmann manifold as a collection of subspaces is fundamental, we often need a more concrete representation. One simple idea is to represent a subspace $S \in \mathcal{G}(k, p)$ by $\boldsymbol{Q} \in \mathcal{V}(k, p)$ having $S$ as its column space. However, the choice of $\boldsymbol{Q}$ is far from unique. Alternatively, the subspace $S$ can be represented uniquely by the orthogonal projection matrix onto $S$, as in Chikuse [7]. We propose instead to represent $\mathcal{G}(k, p)$ by the subset of orthogonal matrices

$$
\mathcal{V}^{+}(k, p)=\left\{\boldsymbol{Q}=\left[\boldsymbol{Q}_{1}^{T} \boldsymbol{Q}_{2}^{T}\right]^{T} \in \mathcal{V}(k, p) \mid \boldsymbol{Q}_{1} \succ \mathbf{0}\right\}
$$

As the next proposition makes precise, almost every element of $\mathcal{G}(k, p)$ can be represented uniquely by an element of $\mathcal{V}^{+}(k, p)$. Recall that we defined $l: \mathcal{V}(k, p) \rightarrow$ $\mathcal{G}(k, p)$ as the map which sends each element of $\mathcal{V}(k, p)$ to its column space.

Proposition 3.2. The map $l$ is injective on $\mathcal{V}^{+}(k, p)$ and the image of $\mathcal{V}^{+}(k, p)$ under $l$ has measure one with respect to the uniform probability measure $P_{\mathcal{G}(k, p)}$ on $\mathcal{G}(k, p)$.

In turn, the set $\mathcal{V}^{+}(k, p)$ is amenable to parametrization by the Cayley transform. In this case, the domain of the Cayley transform $C$ is restricted to the subset

$$
\mathcal{D}_{\mathcal{G}}=\left\{\boldsymbol{X}=\left[\begin{array}{cc}
\mathbf{0} & -\boldsymbol{A}^{T} \\
\boldsymbol{A} & \mathbf{0}_{p-k}
\end{array}\right] \mid \begin{array}{l}
\boldsymbol{A} \in \mathbb{R}^{p-k \times k}, \\
\operatorname{eval}_{i}\left(\boldsymbol{A}^{T} \boldsymbol{A}\right) \in[0,1) \text { for } 1 \leq i \leq k
\end{array}\right\} \subset \operatorname{Skew}(p)
$$

where the notation eval $i\left(\boldsymbol{A}^{T} \boldsymbol{A}\right)$ indicates the $i$ th eigenvalue of the matrix $\boldsymbol{A}^{T} \boldsymbol{A}$. Let $\boldsymbol{X} \in \mathcal{D}_{\mathcal{G}}$ and set $\boldsymbol{Q}=C(\boldsymbol{X})$. Again, partition $\boldsymbol{Q}=\left[\begin{array}{ll}\boldsymbol{Q}_{1}^{T} & \boldsymbol{Q}_{2}^{T}\end{array}\right]^{T}$ so that $\boldsymbol{Q}_{1}$ is square. We can write the blocks of $\boldsymbol{Q}$ in terms of $\boldsymbol{A}$ as

$$
\begin{aligned}
& \boldsymbol{Q}_{1}=\left(\boldsymbol{I}_{k}-\boldsymbol{A}^{T} \boldsymbol{A}\right)\left(\boldsymbol{I}_{k}+\boldsymbol{A}^{T} \boldsymbol{A}\right)^{-1} \\
& \boldsymbol{Q}_{2}=2 \boldsymbol{A}\left(\boldsymbol{I}_{k}+\boldsymbol{A}^{T} \boldsymbol{A}\right)^{-1}
\end{aligned}
$$

We can recover the matrix $\boldsymbol{A}$ from $\boldsymbol{Q}$ :

$$
\boldsymbol{F}=\left(\boldsymbol{I}_{k}-\boldsymbol{Q}_{1}\right)\left(\boldsymbol{I}_{k}+\boldsymbol{Q}_{1}\right)^{-1}
$$




$$
\boldsymbol{A}=\frac{1}{2} \boldsymbol{Q}_{2}\left(\boldsymbol{I}_{k}+\boldsymbol{F}\right)
$$

We turn to verifying the conditions of Section 2. The first two conditions are satisfied as a consequence of the following proposition:

Proposition 3.3. The Cayley transform $C: \mathcal{D}_{\mathcal{G}} \rightarrow \mathcal{G}(k, p)$ is one-to-one.

Only the third condition, that $C$ is continuously differentiable on $\mathcal{D}_{G}$, remains.

The process of computing the derivative matrix is the same as before. We define a $d_{\mathcal{G}}$-dimensional vector $\boldsymbol{\psi}=\operatorname{vec} \boldsymbol{A}$ containing each of the independent entries of $\boldsymbol{X}$. Let $\boldsymbol{X}_{\boldsymbol{\psi}} \in \mathcal{D}_{\mathcal{G}}$ be the matrix having $\boldsymbol{\psi}$ as its corresponding vector of independent entries. The Cayley transform can now be thought of as a function of $\boldsymbol{\psi}$ :

$$
C(\boldsymbol{\psi})=\left(\boldsymbol{I}_{p}+\boldsymbol{X}_{\psi}\right)\left(\boldsymbol{I}_{p}-\boldsymbol{X}_{\psi}\right)^{-1} \boldsymbol{I}_{p \times k}
$$

As a function of $\boldsymbol{\psi}$, the Cayley transform is a bijection between the set $\mathcal{D}_{\mathcal{G}}^{\psi}=\{\boldsymbol{\psi} \in$ $\left.\mathbb{R}^{d_{\mathcal{G}}}: \boldsymbol{X}_{\boldsymbol{\psi}} \in \mathcal{D}_{\mathcal{G}}\right\}$ and $\mathcal{V}^{+}(k, p)$. The next lemma provides an explicit linear map from $\boldsymbol{\psi}$ to vec $\boldsymbol{X}_{\boldsymbol{\psi}}$ in the form of a $\{-1,0,1\}$ matrix $\boldsymbol{\Gamma}_{\mathcal{G}}$ :

Lemma 3.0.2. The equation $\operatorname{vec} X_{\psi}=\boldsymbol{\Gamma}_{\mathcal{G}} \boldsymbol{\psi}$ is satisfied by the matrix

$$
\boldsymbol{\Gamma}_{\mathcal{G}}=\left(\boldsymbol{I}_{p^{2}}-\boldsymbol{K}_{p, p}\right)\left(\boldsymbol{\Theta}_{1}^{T} \otimes \boldsymbol{\Theta}_{2}^{T}\right)
$$

In the next proposition, we identify the derivative matrix $D C(\phi)$.

Proposition 3.4. The Cayley transform is continuously differentiable on $\mathcal{D}_{\mathcal{G}}^{\psi}$ with derivative matrix

$$
D C(\boldsymbol{\psi})=2\left[\boldsymbol{I}_{p \times k}^{T}\left(\boldsymbol{I}_{p}-\boldsymbol{X}_{\psi}\right)^{-T} \otimes\left(\boldsymbol{I}_{p}-\boldsymbol{X}_{\boldsymbol{\psi}}\right)^{-1}\right] \boldsymbol{\Gamma}_{\mathcal{G}}
$$

\section{Change of variables formulas}

We now state change of variables formulas for the Cayley parametrizations of $\mathcal{V}(k, p)$ and $\mathcal{V}^{+}(k, p)$. Given the results of the previous section, the formulas follow directly from Theorem 2.1. The $d_{\mathcal{\nu}}$-dimensional Jacobian $J_{d_{\mathcal{V}}} C(\boldsymbol{\varphi})$ of the Cayley transform $C$ at $\varphi$ is equal to

$$
J_{d_{\mathcal{V}}} C(\boldsymbol{\varphi})=\left|D C(\boldsymbol{\varphi})^{T} D C(\boldsymbol{\varphi})\right|^{1 / 2}
$$




$$
=\left|2^{2} \boldsymbol{\Gamma}_{\mathcal{V}}^{T}\left(\boldsymbol{G}_{\mathcal{V}} \otimes \boldsymbol{H}_{\mathcal{V}}\right) \boldsymbol{\Gamma}_{\mathcal{V}}\right|^{1 / 2}
$$

where

$$
\begin{aligned}
& \boldsymbol{G}_{\mathcal{V}}=\left(\boldsymbol{I}_{p}-\boldsymbol{X}_{\boldsymbol{\varphi}}\right)^{-1} \boldsymbol{I}_{p \times k} \boldsymbol{I}_{p \times k}^{T}\left(\boldsymbol{I}_{p}-\boldsymbol{X}_{\boldsymbol{\varphi}}\right)^{-T} \\
& \boldsymbol{H}_{\mathcal{V}}=\left(\boldsymbol{I}_{p}-\boldsymbol{X}_{\boldsymbol{\varphi}}\right)^{-T}\left(\boldsymbol{I}_{p}-\boldsymbol{X}_{\boldsymbol{\varphi}}\right)^{-1}
\end{aligned}
$$

and $\boldsymbol{\Gamma}_{\mathcal{V}}$ is defined as in Section 3.1. The equations above hold for the $d_{\mathcal{G}}$-dimensional Jacobian $J_{d_{\mathcal{G}}} C(\phi)$ if we replace the symbols $\mathcal{V}$ and $\varphi$ with the symbols $\mathcal{G}$ and $\boldsymbol{\psi}$, respectively. In the supplementary material, we describe how to compute the Jacobian terms, taking advantage of their block structure. Let $g: \mathcal{V}(k, p) \rightarrow \mathbb{R}$ be proportional to a density with respect to the $d_{\mathcal{V}}$-dimensional Hausdorff measure on $\mathcal{V}(k, p)$.

Theorem 4.1. (Change of Variables Formulas) For all Borel sets $A \subset \mathcal{D}_{\mathcal{V}}^{\varphi}$

$$
\int_{A} J_{d_{\mathcal{V}}} C(\boldsymbol{\varphi}) L^{d_{\mathcal{V}}}(d \boldsymbol{\varphi})=H^{d_{\mathcal{V}}}[C(A)]
$$

and hence

$$
\int_{A} g[C(\boldsymbol{\varphi})] J_{d_{\mathcal{V}}} C(\boldsymbol{\varphi}) L^{d_{\mathcal{V}}}(d \boldsymbol{\varphi})=\int_{C(A)} g(\boldsymbol{Q}) H^{d_{\mathcal{V}}}(d \boldsymbol{Q})
$$

If instead we have $g: \mathcal{V}^{+}(k, p) \rightarrow \mathbb{R}$ proportional to a density with respect to the $d_{\mathcal{G}^{-}}$ dimensional Hausdorff measure on $\mathcal{V}^{+}(k, p)$, the statement is true when we replace the symbols $\mathcal{V}$ and $\boldsymbol{\varphi}$ with the symbols $\mathcal{G}$ and $\boldsymbol{\psi}$, respectively.

Similarly to Theorem 2.1, Theorem 4.1 has an interpretation in terms of random variables. Let the distribution of $\boldsymbol{Q} \in \mathcal{V}(k, p)$ have a density proportional to $g$. Then $\boldsymbol{Q} \stackrel{\text { dist. }}{=} C(\varphi)$ when the distribution of $\varphi \in \mathcal{D}_{\mathcal{V}}^{\varphi}=\mathbb{R}^{d_{\mathcal{V}}}$ has a density proportional to $g[C(\boldsymbol{\varphi})] J_{d_{\mathcal{V}}} C(\boldsymbol{\varphi})$. In particular, let $g \propto 1$ so that $\boldsymbol{Q} \sim P_{\mathcal{V}(k, p)}$. Then $\boldsymbol{Q} \stackrel{\text { dist. }}{=}$ $C(\varphi)$ when the distribution of $\varphi$ has a density proportional to $J_{d_{\mathcal{V}}} C(\boldsymbol{\varphi})$. Analogous statements hold when $\boldsymbol{Q}$ is a random element of $\mathcal{V}^{+}(k, p$. $)$

\section{$5 \quad$ Simulating from $\mathcal{V}(k, p)$ and $\mathcal{V}^{+}(k, p)$}

Practical applications often require simulating a random orthogonal matrix $\boldsymbol{Q}$ whose distribution has a prescribed density $g$. For instance, Bayesian analyses of statistical 
models with an orthogonal matrix parameter yield posterior densities on the Stiefel manifold, and inference typically requires simulating from these densities. In many cases, generating independent samples is too challenging and MCMC methods are the most sensible option.

In this section, we present an MCMC approach to simulating from a distribution having a density on the set $\mathcal{V}(k, p)$ or $\mathcal{V}^{+}(k, p)$ which takes advantage of the Cayley parametrizations described in Section 3. The recent work of Pourzanjani et al. [38] explores a similar idea based on a Givens rotation parametrization of the Stiefel manifold. When it is not too computationally expensive, our approach may have certain advantages over existing methods. Unlike Hoff [19], it can be applied regardless of whether conditional distributions belong to a particular parametric family, and it is arguably simpler to implement than the approach of Byrne and Girolami [3]. In statistical applications where interest lies in a subspace rather than a particular orthogonal basis, our representation of the Grassmann manifold $\mathcal{G}(k, p)$ by the set $\mathcal{V}^{+}(k, p)$ may suggest an appealing parametrization, with the MCMC approach of this section offering the machinery for Bayesian inference.

We illustrate the basic idea with the Stiefel manifold. (Simulating from $\mathcal{V}^{+}(k, p)$ involves analogous steps.) In order to simulate $\boldsymbol{Q}$ whose distribution has density $g$ on the Stiefel manifold $\mathcal{V}(k, p)$, we construct a Markov chain whose stationary distribution has density $g[C(\varphi)] J_{d_{\mathcal{V}}} C(\varphi)$ on the set $\mathcal{D}_{\mathcal{V}}^{\varphi}=\mathbb{R}^{d_{\mathcal{V}}}$. Then we simply transform the realized Markov chain back to $\mathcal{V}(k, p)$ using the Cayley transform. By doing so, we avoid the difficulty of choosing and simulating from an efficient proposal distribution defined on the Stiefel manifold.

To make things more concrete, we describe the approach with the MetropolisHastings algorithm as our MCMC method. We start with an initial value $\varphi_{0} \in \mathcal{D}_{\mathcal{V}}^{\varphi}$ for our chain and a density $q\left(\varphi^{\prime} \mid \varphi\right)$ for the proposal $\varphi^{\prime}$ given the previous value $\varphi$. The Metropolis-Hastings algorithm for simulating from the distribution having density $g[C(\boldsymbol{\varphi})] J_{d_{\mathcal{V}}} C(\boldsymbol{\varphi})$ on $\mathcal{D}_{\mathcal{V}}^{\varphi}$ proceeds as follows. For $t=0, \ldots, T$ :

1. Generate $\varphi^{\prime}$ from $q\left(\varphi^{\prime} \mid \varphi_{t}\right)$.

2. Compute the acceptance ratio

$$
r=\frac{g\left[C\left(\boldsymbol{\varphi}^{\prime}\right)\right] J_{d_{\nu}} C\left(\boldsymbol{\varphi}^{\prime}\right)}{g\left[C\left(\boldsymbol{\varphi}_{t}\right)\right] J_{d_{\mathcal{\nu}}} C\left(\boldsymbol{\varphi}_{t}\right)} \frac{q\left(\boldsymbol{\varphi}_{t} \mid \boldsymbol{\varphi}^{\prime}\right)}{q\left(\boldsymbol{\varphi}^{\prime} \mid \boldsymbol{\varphi}_{t}\right)}
$$

3. Sample $u \sim \operatorname{Unif}(0,1)$. If $u \leq r$, set $\varphi_{t+1}=\varphi^{\prime}$. Otherwise, set $\varphi_{t+1}=\varphi_{t}$. 

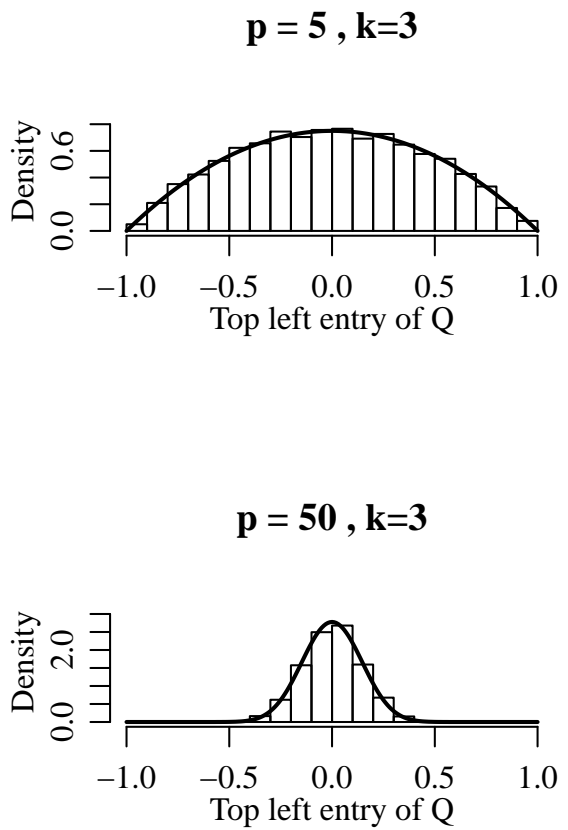

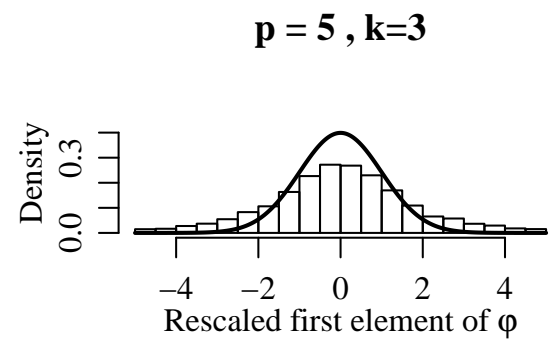

$\mathbf{p}=\mathbf{5 0}, \mathbf{k}=\mathbf{3}$

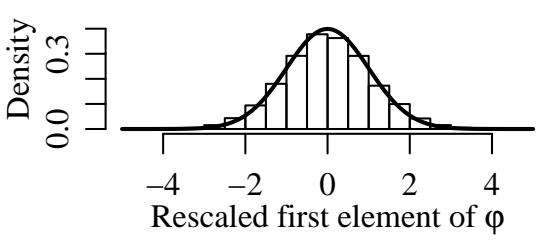

Figure 1: The top left panel is a histogram of the top left entry of simulated values of $\boldsymbol{Q} \sim P_{\mathcal{V}(3,5)}$ plotted against the exact density. The bottom left panel is the analogous plot for $\boldsymbol{Q} \sim P_{\mathcal{V}(3,50)}$. The top right panel is a histogram of the first entry, rescaled by $\sqrt{p / 2}$, of $\boldsymbol{\varphi}=C^{-1}(\boldsymbol{Q})$ when $p=5$ and $k=3$. The histogram is plotted against a standard normal density. The bottom right panel is the analogous plot for the case when $p=50$ and $k=3$.

For a broad class of $g$ and $q$, the orthogonal matrices $\left\{C\left(\boldsymbol{\varphi}_{t}\right)\right\}_{t=0}^{T}$ approximate the distribution having density $g$ when $T$ is large enough.

In place of this simple Metropolis-Hastings algorithm, we can substitute other MCMC methods. Hamiltonian Monte Carlo (HMC) [36], with implementations in software such as Carpenter et al. [4] and Salvatier et al. [41], is a natural choice. For settings in which evaluation and automatic differentiation of the Jacobian term are not prohibitively expensive, the Cayley transform approach offers a relatively straightforward path to MCMC simulation on the sets $\mathcal{V}(k, p)$ and $\mathcal{V}^{+}(k, p)$. 


\subsection{Example: The uniform distribution on $\mathcal{V}(k, p)$}

Using the MCMC approach described above, we simulate from the uniform distribution on $\mathcal{V}(k, p)$. Specifically, we use HMC as implemented in Stan [4] to simulate $\boldsymbol{Q} \sim P_{\mathcal{V}(k, p)}$. Of course, there exist many algorithms for the simulation of independent, uniformly distributed orthogonal matrices. The uniform distribution only serves as a starting point for illustrating the proposed approach.

Figure 1 provides plots based on 10,000 simulated values of $\boldsymbol{Q} \sim P_{\mathcal{V}(k, p)}$. The top row of the figure deals with the case $p=5$ and $k=3$, while the bottom row deals with the case $p=50$ and $k=3$. The histograms on the left show the top left entry of the simulated values of $\boldsymbol{Q}$ plotted against the exact density, given in Proposition 7.3 of Eaton [17]. As we expect, there is close agreement between the histogram density estimate and the exact density. The histograms on the right show the first entry, rescaled by $\sqrt{p / 2}$, of the simulated values of the vector $\varphi=C^{-1}(Q)$. These are plotted against a standard normal density. Theorem 6.1 tells us that the histogram density estimate and the standard normal density should agree when $p$ is large (both in an absolute sense and relative to $\mathrm{k}$ ), which is what we observe in the plot on the bottom right. When $k$ is similar in magnitude to $p$, the standard normal density is a poor approximation, as we see in the plot on the top right.

\subsection{Example: Bayesian inference for the spiked covariance model}

Suppose the rows of an $n \times p$ data matrix $\boldsymbol{Y}$ are independent samples from a mean zero multivariate normal population with covariance matrix $\boldsymbol{\Sigma}$. The spiked covariance model, considered by Johnstone [26] and others, assumes the covariance matrix $\boldsymbol{\Sigma}$ can be decomposed as $\boldsymbol{\Sigma}=\sigma^{2}\left(\boldsymbol{Q} \boldsymbol{\Lambda} \boldsymbol{Q}^{T}+\boldsymbol{I}_{p}\right)$ with $\boldsymbol{Q} \in \mathcal{V}(k, p)$ and $\boldsymbol{\Lambda}=\operatorname{diag}\left(\lambda_{1}, \ldots, \lambda_{k}\right)$ where $\lambda_{1}>\ldots>\lambda_{k}>0$. Under this model, the covariance matrix is partitioned into the low rank "signal" component $\sigma^{2} \boldsymbol{Q} \boldsymbol{\Lambda} \boldsymbol{Q}^{T}$ and the isotropic "noise" component $\sigma^{2} \boldsymbol{I}_{p}$.

Given priors for the unknown parameters, a conventional Bayesian analysis will approximate the posterior distribution by a Markov chain having the posterior as its stationary distribution. Inference for the trivially constrained parameters $\sigma^{2}$ and $\boldsymbol{\Lambda}$ is easily handled by standard MCMC approaches, so we treat these parameters as fixed and focus on inference for the orthogonal matrix parameter $\boldsymbol{Q}$. With a uniform 

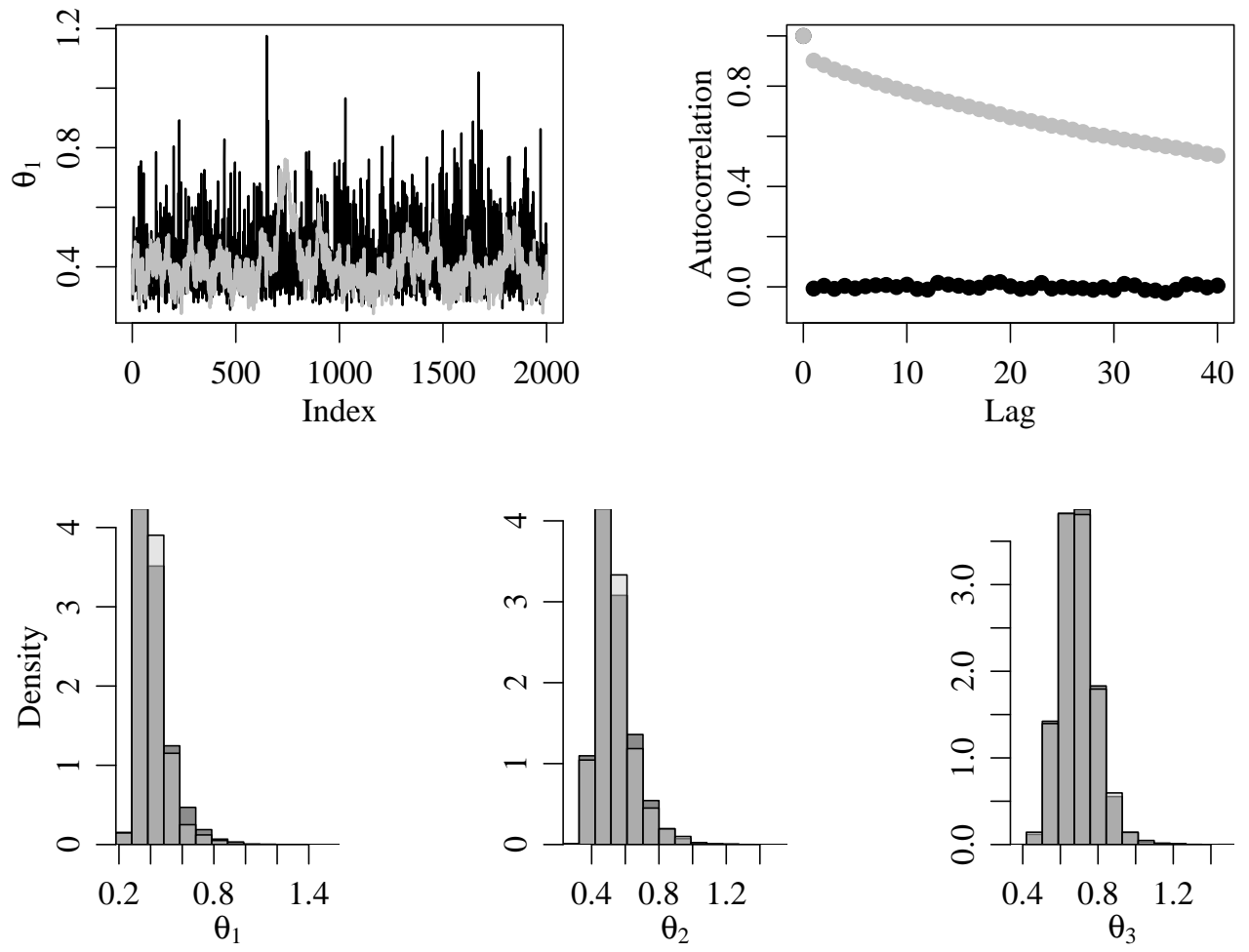

Figure 2: The plot in the top left overlays trace plots of the first principal angle calculated from a portion of each of the chains, while the plot in the top right shows the correlation between lagged values of the first principal angle. The black lines and dots correspond to our MCMC approach, while the gray lines and dots correspond to that of Hoff [19]. The plots in the bottom half compare histogram approximations of the marginal posterior distributions of the principal angles. 
prior for $\boldsymbol{Q}$, the posterior distribution is matrix Bingham [19], having density

$$
p\left(\boldsymbol{Q} \mid \boldsymbol{Y}, \sigma^{2}, \boldsymbol{\Lambda}\right) \propto \operatorname{etr}\left\{\left[\left(\boldsymbol{\Lambda}^{-1}+\boldsymbol{I}_{k}\right)^{-1} /\left(2 \sigma^{2}\right)\right] \boldsymbol{Q}^{T}\left[\boldsymbol{Y}^{T} \boldsymbol{Y}\right] \boldsymbol{Q}\right\} .
$$

We compare two MCMC approaches to simulating from the matrix Bingham distribution: the Gibbs sampling method of Hoff [19] and the Cayley transform approach (again, with HMC as implemented in Stan). The dimensions are chosen as $n=100, p=50$, and $k=3$. We set $\sigma^{2}=1, \boldsymbol{\Lambda}=\operatorname{diag}(5,3,1.5)$, and choose a true value of $\boldsymbol{Q}$ uniformly from $\mathcal{V}(3,50)$. We then generate a data matrix according to the model vec $\boldsymbol{Y} \sim \mathcal{N}\left[\mathbf{0}, \sigma^{2}\left(\boldsymbol{Q} \boldsymbol{\Lambda} \boldsymbol{Q}^{T}+\boldsymbol{I}_{p}\right) \otimes \boldsymbol{I}_{100}\right]$. We run each Markov chain for 12,000 steps and discard the first 2000 steps as burn-in. In order to summarize the high-dimensional posterior simulations in terms of lower dimensional quantities, we compute the principal angles between the columns of the simulated $\boldsymbol{Q}$ matrices and the corresponding columns of the posterior mode $\boldsymbol{V}$, computed from the eigendecomposition $\boldsymbol{A}=\boldsymbol{V} \boldsymbol{D} \boldsymbol{V}^{T}$. For $j=1, \ldots, 3$, the principal angles are

$$
\theta_{j}=\cos ^{-1}\left(\frac{\left|\boldsymbol{q}_{j}^{T} \boldsymbol{v}_{j}\right|}{\left\|\boldsymbol{q}_{j}\right\|\left\|\boldsymbol{v}_{j}\right\|}\right)
$$

where $\boldsymbol{q}_{j}$ and $\boldsymbol{v}_{j}$ are the $j$ th columns of $\boldsymbol{Q}$ and $\boldsymbol{V}$, respectively.

Figure 2 displays the principal angles calculated from the two Markov chains. The plots in the bottom half of the figure compare histogram approximations of the marginal posterior distributions of the principal angles. There is considerable overlap, suggesting that the two chains have found their way to equivalent modes of their matrix Bingham stationary distribution. The plot in the top left of the figure overlays trace plots of the first principal angle calculated from a portion of each of the chains. The black line corresponds to our MCMC approach, while the gray line corresponds to that of Hoff [19]. The plot in the top right shows the correlation between lagged values of the first principal angle. Again, the black dots correspond to our MCMC approach, while the gray dots correspond to that of Hoff [19]. Together, the plots in the top half of the figure indicate that, compared to the approach of Hoff [19], the Cayley transform approach produces a Markov chain with less autocorrelation, reducing Monte Carlo error in the resulting posterior inferences. 


\section{An asymptotic independent normal approximation}

In Section 4, we derived the density for the distribution of $\boldsymbol{\varphi}=C^{-1}(\boldsymbol{Q})$ when $\boldsymbol{Q}$ is distributed uniformly on the Stiefel manifold. However, the expression involves the rather opaque Jacobian term $J_{d_{\mathcal{V}}} C(\varphi)$. Instead of analyzing the density function, we can gain insight into the distribution of $\varphi$ by other means. The critical observation, evident in simulations, is the following: If $\boldsymbol{Q} \sim P_{\mathcal{V}(k, p)}$ with $p$ large and $p \gg k$ then, in some sense, the elements of $\boldsymbol{\varphi}=C^{-1}(\boldsymbol{Q})$ are approximately independent and normally distributed. Theorem 6.1 of this section provides a mathematical explanation for this empirical phenomenon.

In order to understand Theorem 6.1 and its broader context, it is helpful to review the literature relating to normal approximations to the entries of random orthogonal matrices or transformations thereof. For the sake of consistency and clarity, the notation and formulation of the relevant results have been modified slightly. Let $\left\{\boldsymbol{Q}_{p}\right\}$ be a sequence of random orthogonal matrices with each element $\boldsymbol{Q}_{p}$ uniform on $\mathcal{V}\left(k_{p}, p\right)$. The notation $k_{p}$ indicates that the number of columns may grow with the number of rows. For each $p$, let $q_{p}$ be the top left entry of $\boldsymbol{Q}_{p}$ (any other entry would also work). It has long been observed that $q_{p}$ is approximately normal when $p$ is large. The earliest work in this direction relates to the equivalence of ensembles in statistical mechanics and is due to Mehler [35], Maxwell [32, 33], and Borel [2]. A theorem of Borel shows that $\operatorname{Pr}\left(\sqrt{p} q_{p} \leq x\right) \rightarrow \Phi(x)$ as $p$ grows, where $\Phi$ is the cumulative distribution function of a standard normal random variable. Since then, a large and growing literature on this sort of normal approximation has emerged. A detailed history is given in Diaconis and Freedman [12], while an overview is given in D'Aristotile et al. [10] and Jiang [23].

Much of this literature is devoted to normal approximations to the joint distribution of entries of random orthogonal matrices. Letting $\boldsymbol{q}_{p}$ be the first column of $\boldsymbol{Q}_{p}$ for each $p$ (again, any other column would also work), Stam [43] proved that the total variation distance between the distribution of the first $m_{p}$ coordinates of $\boldsymbol{q}_{p}$ and the distribution of $m_{p}$ standard normal random variables converges to zero as $p$ gets large so long as $m_{p}=o(\sqrt{p})$. Diaconis and Freedman [12] strengthened this result, showing that it holds for $m_{p}=o(p)$. Diaconis et al. [14] prove that the total variation distance between the distribution of the top left $m_{p} \times n_{p}$ block of $\boldsymbol{Q}_{p}$ and the distribution of $m_{p} n_{p}$ independent standard normals goes to zero as $p \rightarrow \infty$ if $m_{p}=o\left(p^{\gamma}\right)$ and $n_{p}=o\left(p^{\gamma}\right)$ for $\gamma=1 / 3$. (Clearly, we must have $n_{p} \leq k_{p}$ for this 
result to make sense.) Their work drew attention to the problem of determining the largest orders of $m_{p}$ and $n_{p}$ such that the total variation distance goes to zero. Jiang [23] solved this problem, finding the largest orders to be $o\left(p^{1 / 2}\right)$. Recent work has further explored this topic [45, 24].

Many authors have also considered transformations of random orthogonal matrices or notions of approximation not based on total variation distance. In the former category, D'Aristotile et al. [10] and Meckes [34] study the convergence of linear combinations of the entries of the matrices in the sequence $\left\{\boldsymbol{Q}_{p}\right\}$ to normality as $p \rightarrow \infty$. Diaconis and Shahshahani [13], Stein [44], Johansson [25], and Rains [39] addresses the normality of traces of powers of random orthogonal and unitary matrices. In the latter category, Chatterjee and Meckes [6] and Jiang and Ma [24] consider probability metrics other than total variation distance. Jiang [23] also considers a notion of approximation other than total variation distance, and Theorem 3 in that work is particularly important in understanding our Theorem 6.1.

Theorem 3 of Jiang [23] tells us that the distribution $P_{\mathcal{V}\left(k_{p}, p\right)}$ can be approximated by the distribution of $p k_{p}$ independent normals provided that $p$ is sufficiently large (both in an absolute sense and relative to $k_{p}$ ). The form of approximation in the theorem is weaker and likely less familiar than one based on total variation distance. Define the max norm $\|\cdot\|_{\max }$ of a matrix as the maximum of the absolute values of its entries. Jiang [23] shows that one can construct a sequence of pairs of random matrices $\left\{\boldsymbol{Z}_{p}, \boldsymbol{Q}_{p}\right\}$ with each pair defined on the same probability space such that

(i) The entries of the $p \times k_{p}$ matrix $\boldsymbol{Z}_{p}$ are independent standard normals;

(ii) The matrix $\boldsymbol{Q}_{p}$ is uniform on $\mathcal{V}\left(k_{p}, p\right)$;

(iii) The quantity $\epsilon_{p}=\left\|\sqrt{p} \boldsymbol{Q}_{p}-\boldsymbol{Z}_{p}\right\|_{\max } \rightarrow 0$ in probability as $p$ grows provided that $k_{p}=o(p / \log p)$, and this is the largest order of $k_{p}$ such that the result holds.

The coupling is constructed by letting $\boldsymbol{Q}_{p}$ be the result of the Gram-Schmidt orthogonalization procedure applied to $\boldsymbol{Z}_{p}$.

To better understand this type of approximation, which we refer to as 'approximation in probability,' consider the problem of simulating from the distribution of the random matrix $\sqrt{p} \boldsymbol{Q}_{p}$ on a computer with finite precision. One could simulate a matrix $\boldsymbol{Z}_{p}$ of independent standard normals, obtain $\boldsymbol{Q}_{p}$ using Gram-Schmidt, then 
multiply by $\sqrt{p}$ to arrive at $\sqrt{p} \boldsymbol{Q}_{p}$. However, for a fixed machine precision and $p$ sufficiently large (again, both in an absolute sense and relative to $k_{p}$ ), the matrix $\sqrt{p} \boldsymbol{Q}_{p}$ would be indistinguishable from $\boldsymbol{Z}_{p}$ with high probability.

Our Theorem 6.1 establishes that the distribution of $\boldsymbol{\varphi}_{p}=C_{p}^{-1}\left(\boldsymbol{Q}_{p}\right)$, which we know to have a density proportional to $J_{d_{\mathcal{V}}} C_{p}(\boldsymbol{\varphi})$, can be approximated in probability by independent normals. (Since we now have a sequence of matrices of different dimensions, we denote the Cayley transform parametrizing $\mathcal{V}\left(k_{p}, p\right)$ by $C_{p}$.) For each $p$, define the diagonal scale matrix

$$
\boldsymbol{\Pi}_{p}=\left[\begin{array}{cc}
\sqrt{p / 2} \boldsymbol{I}_{k_{p}\left(k_{p}-1\right) / 2} & \mathbf{0} \\
\mathbf{0} & \sqrt{p} \boldsymbol{I}_{\left(p-k_{p}\right) k_{p}}
\end{array}\right],
$$

and recall that the infinity norm $\|\cdot\|_{\infty}$ of a vector is equal to the maximum of the absolute values of its entries.

Theorem 6.1. One can construct a sequence of pairs of random vectors $\left\{\boldsymbol{z}_{p}, \boldsymbol{\varphi}_{p}\right\}$ such that

(i) The entries of the vector $\boldsymbol{z}_{p}$ are independent standard normals;

(ii) The vector $\boldsymbol{\varphi}_{p} \stackrel{\text { dist. }}{=} C_{p}^{-1}\left(\boldsymbol{Q}_{p}\right)$ where $\boldsymbol{Q}_{p} \sim P_{\mathcal{V}\left(k_{p}, p\right)}$;

(iii) The quantity $\epsilon_{p}:=\left\|\boldsymbol{\Pi}_{p} \boldsymbol{\varphi}_{p}-\boldsymbol{z}_{p}\right\|_{\infty} \rightarrow 0$ in probability as $p \rightarrow \infty$ provided $k_{p}=o\left(\frac{p^{1 / 4}}{\sqrt{\log p}}\right)$.

The construction of the coupling is more elaborate than in Theorem 3 of Jiang [23]. We first introduce a function $\widetilde{C}_{p}^{-1}$ which approximates the inverse Cayley transform. Given a matrix $\boldsymbol{M} \in \mathbb{R}^{p \times k_{p}}$ having a square top block $\boldsymbol{M}_{1}$ and a bottom block $\boldsymbol{M}_{2}$, the vector $\widetilde{b}_{p}(\boldsymbol{M})$ contains the independent entries of $\widetilde{B}_{p}(\boldsymbol{M})=\boldsymbol{M}_{1}-\boldsymbol{M}_{1}^{T}$ obtained by eliminating diagonal and supradiagonal entries from vec $\widetilde{B}_{p}(\boldsymbol{M})$ while $\widetilde{A}_{p}(\boldsymbol{M})=\boldsymbol{M}_{2}$. The approximate inverse Cayley transform is then

$$
\widetilde{C}_{p}^{-1}(\boldsymbol{M})=\left[\begin{array}{c}
\widetilde{b}_{p}(\boldsymbol{M}) \\
\operatorname{vec} \widetilde{A}_{p}(\boldsymbol{M})
\end{array}\right] .
$$

Now let $\boldsymbol{Z}_{p}$ be a $p \times k_{p}$ matrix of independent standard normals and let $\boldsymbol{Q}_{p}$ be the result of applying the Gram-Schmidt orthogonalization procedure to $\boldsymbol{Z}_{p}$. It follows that $\boldsymbol{Q}_{p} \sim P_{\mathcal{V}\left(k_{p}, p\right)}$. Finally, set

$$
\boldsymbol{z}_{p}=\boldsymbol{\Pi}_{p} \widetilde{C}_{p}^{-1}\left(p^{-1 / 2} \boldsymbol{Z}_{p}\right)
$$




$$
\boldsymbol{\varphi}_{p}=C_{p}^{-1}\left(\boldsymbol{Q}_{p}\right)
$$

The details of the proof of Theorem 6.1 appear in the appendix, but we provide a sketch here. Part (i) is straightforward to verify and (ii) is immediate. Part (iii) requires more work. The proof of (iii) involves verifying the following proposition, which involves a third random vector $\widetilde{\varphi}_{p}=\widetilde{C}_{p}^{-1}\left(\boldsymbol{Q}_{p}\right)$ :

Proposition 6.1. (i) The quantity $\left\|\boldsymbol{\Pi}_{p} \widetilde{\boldsymbol{\varphi}}_{p}-\boldsymbol{\Pi}_{p} \boldsymbol{\varphi}_{p}\right\|_{\infty} \rightarrow 0$ in probability as $p \rightarrow \infty$ provided $k_{p}=o\left(\frac{p^{1 / 4}}{\sqrt{\log p}}\right)$, and (ii) the quantity $\left\|\boldsymbol{\Pi}_{p} \widetilde{\boldsymbol{\varphi}}_{p}-\boldsymbol{z}_{p}\right\|_{\infty} \rightarrow 0$ in probability as $p \rightarrow \infty$ provided $k_{p}=o\left(\frac{p}{\log p}\right)$.

Part (iii) then follows from the proposition by the triangle inequality.

\section{Acknowledgements}

This work was partially supported by grants from the National Science Foundation (DMS-1505136, IIS-1546130) and the United States Office of Naval Research (N00014-14-1-0245/N00014-16-1-2147).

\section{References}

[1] T. W. Anderson, I. Olkin, and L. G. Underhill. Generation of Random Orthogonal Matrices. SIAM Journal on Scientific and Statistical Computing, 8 (4):625-629, 1987.

[2] Émile Borel. Sur les principes de la théorie cinétique des gaz. Annales scientifiques de l'École Normale Supérieure, 23:9-32, 1906.

[3] Simon Byrne and Mark Girolami. Geodesic Monte Carlo on embedded manifolds. Scandinavian Journal of Statistics, 40(4):825-845, 2013.

[4] Bob Carpenter, Andrew Gelman, Matthew D. Hoffman, Daniel Lee, Ben Goodrich, Michael Betancourt, Marcus Brubaker, Jiqiang Guo, Peter Li, and Allen Riddell. Stan : A Probabilistic Programming Language. Journal of Statistical Software, 76(1):1-32, 2017.

[5] Arthur Cayley. Sur quelques propriétés des déterminants gauches. Journal für die reine und angewandte Mathematik, 32:119-123, 1846. 
[6] Sourav Chatterjee and Elizabeth Meckes. Multivariate normal approximation using exchangeable pairs. Latin American Journal of Probability and Mathematical Statistics, 4:257-283, 2008.

[7] Yasuko Chikuse. Statistics on Special Manifolds. Springer New York, 2003.

[8] R. Dennis Cook, Bing Li, and Francesca Chiaromonte. Envelope models for parsimonious and efficient multivariate linear regression. Statistica Sinica, 20 (3):927-960, 2010.

[9] Andrew Cron and Mike West. Models of Random Sparse Eigenmatrices and Bayesian Analysis of Multivariate Structure. In Statistical Analysis for HighDimensional Data, pages 125-153. Springer International Publishing, 2016.

[10] Anthony D'Aristotile, Persi Diaconis, and Charles M. Newman. Brownian motion and the classical groups. Lecture Notes-Monograph Series, 41:97-116, 2003.

[11] Persi Diaconis and Peter J. Forrester. Hurwitz and the origins of random matrix theory in mathematics. Random Matrices: Theory and Applications, 6(1), 2017.

[12] Persi Diaconis and David Freedman. A dozen de Finetti-style results in search of a theory. Annales de l'Institut Henri Poincaré, Probabilités et Statistiques, 23(2):397-423, 1987.

[13] Persi Diaconis and Mehrdad Shahshahani. On the Eigenvalues of Random Matrices. Journal of Applied Probability, 31:49-62, 1994.

[14] Persi Diaconis, Morris Eaton, and Steffen Lauritzen. Finite De Finetti Theorems in Linear Models and Multivariate Analysis. Scandinavian Journal of Statistics, 19(4):289-315, 1992.

[15] Persi Diaconis, Susan Holmes, and Mehrdad Shahshahani. Sampling from a Manifold. In Advances in Modern Statistical Theory and Applications: A Festschrift in honor of Morris L. Eaton, pages 102-125. Institute of Mathematical Statistics, 2013.

[16] Morris L. Eaton. Multivariate Statistics: a Vector Space Approach. John Wiley \& Sons, 1983. 
[17] Morris L. Eaton. Group Invariance Applications in Statistics. In Regional Conference Series in Probability and Statistics, volume 1, pages i-133. Institute of Mathematical Statistics, 1989.

[18] Peter D. Hoff. Model Averaging and Dimension Selection for the Singular Value Decomposition. Journal of the American Statistical Association, 102(478):674$685,2007$.

[19] Peter D. Hoff. Simulation of the matrix Bingham-von Mises-Fisher Distribution, With Applications to Multivariate and Relational Data. Journal of Computational and Graphical Statistics, 18(2):438-456, 2009.

[20] J. H. Hubbard and B. B. Hubbard. Vector Calculus, Linear Algebra, and Differential Forms: A Unified Approach. Matrix Editions, 2009.

[21] A. Hurwitz. Über die Erzeugung der invarianten durch integration. Nachrichten von der Gesellschaft der Wissenschaften zu Göttingen, MathematischPhysikalische Klasse, 1897:71-90, 1897.

[22] A. T. James. Normal Multivariate Analysis and the Orthogonal Group. Annals of Mathematical Statistics, 25(1):40-75, 1954.

[23] Tiefeng Jiang. How many entries of a typical orthogonal matrix can be approximated by independent normals? Annals of Probability, 34(4):1497-1529, 2006 .

[24] Tiefeng Jiang and Yutao Ma. Distances between random orthogonal matrices and independent normals. arXiv:1704.05205v1, 2017.

[25] Kurt Johansson. On Random Matrices from the Compact Classical Groups. Annals of Mathematics, 145(3):519-545, 1997.

[26] Iain M. Johnstone. On the distribution of the largest eigenvalue in principal components analysis. The Annals of Statistics, 29(2):295-327, 2001.

[27] Carlos A. León, Jean-Claude Massé, and Louis-Paul Rivest. A Statistical Model for Random Rotations. Journal of Multivariate Analysis, 97(2):412-430, 2006.

[28] Jan R. Magnus. Linear Structures. Oxford University Press, 1988. 
[29] Jan R. Magnus and H. Neudecker. The Commutation Matrix: Some Properties and Applications. The Annals of Statistics, 7(2):381-394, 1979.

[30] Jan R. Magnus and Heinz Neudecker. Matrix differential calculus with applications in statistics and econometrics. Wiley Series in Probability and Mathematical Statistics, 1988.

[31] K. V. Mardia and P. E. Jupp. Directional Statistics. Wiley Series in Probability and Statistics, 2009.

[32] J. C. Maxwell. Theory of Heat. Longmans, London, 4th edition, 1875.

[33] J. C. Maxwell. On Boltzmann's theorem on the average distribution of energy in a system of material points. Transactions of the Cambridge Philosophical Society, 12:547-575, 1878.

[34] Elizabeth Meckes. Linear functions on the classical matrix groups. Transactions of the American Mathematical Society, 360(10):5355-5366, 2008.

[35] F. G. Mehler. Ueber die Entwicklung einer Function von beliebig vielen. Variablen nach Laplaschen Functionen höherer Ordnung. Crelle's Journal, 66:161$176,1866$.

[36] Radford M. Neal. MCMC Using Hamiltonian Dynamics. Handbook of Markov Chain Monte Carlo, 54:113-162, 2010.

[37] H. Neudecker. On Jacobians of transformations with skew-symmetric, strictly (lower) triangular or diagonal matrix arguments. Linear and Multilinear Algebra, 14(3):271-295, 1983.

[38] Arya A. Pourzanjani, Richard M. Jiang, Brian Mitchell, Paul J. Atzberger, and Linda R. Petzold. General Bayesian Inference over the Stiefel Manifold via the Givens Transform. arXiv:1710.09443v2, 2017.

[39] E. M. Rains. High powers of random elements of compact Lie groups. Probability Theory and Related Fields, 107(2):219-241, 1997.

[40] Vinayak Rao, Lizhen Lin, and David B. Dunson. Data augmentation for models based on rejection sampling. Biometrika, 103(2):319-335, 2016. 
[41] John Salvatier, Thomas V. Wiecki, and Christopher Fonnesbeck. Probabilistic programming in Python using PyMC3. PeerJ Computer Science, 2:e55, 2016.

[42] Ron Shepard, Scott R. Brozell, and Gergely Gidofalvi. The Representation and Parametrization of Orthogonal Matrices. The Journal of Physical Chemistry A, 119(28):7924-7939, 2015.

[43] A. J. Stam. Limit theorems for uniform distributions on spheres in highdimensional Euclidean spaces. Journal of Applied Probability, 19:221-228, 1982.

[44] C. Stein. The accuracy of the normal approximation to the distribution of the traces of powers of random orthogonal matrices. Technical Report No. 470, Stanford University Department of Statistics, 1995.

[45] Kathryn Stewart. Total variation approximation of random orthogonal matrices by Gaussian matrices. arXiv:1704.06641v2, 2018.

[46] Tim Traynor. Change of variable for Hausdorff measure. Rendiconti dell'Istituto di Matematica dell'Università di Trieste, 1:327-347, 1993.

[47] Ramon van Handel. Probability in high dimension. Technical report, Princeton University, 2016.

\section{A Proofs}

\section{A.1 The sum of a symmetric positive definite matrix and skew- symmetric matrix is nonsingular}

Let $\boldsymbol{\Sigma}$ and $\boldsymbol{S}$ be symmetric positive definite and skew-symmetric, respectively, of equal dimension. Their sum can be written

$$
\boldsymbol{\Sigma}+\boldsymbol{S}=\boldsymbol{\Sigma}^{1 / 2}\left(I+\boldsymbol{\Sigma}^{-1 / 2} \boldsymbol{S} \boldsymbol{\Sigma}^{-1 / 2}\right) \boldsymbol{\Sigma}^{1 / 2}
$$

The matrix $\boldsymbol{\Sigma}^{-1 / 2} \boldsymbol{S} \boldsymbol{\Sigma}^{-1 / 2}$ is skew-symmetric, which implies that $\boldsymbol{I}+\boldsymbol{\Sigma}^{-1 / 2} \boldsymbol{S} \boldsymbol{\Sigma}^{-1 / 2}$ is nonsingular. Because it can be written as the product of nonsingular matrices, the sum $\boldsymbol{\Sigma}+\boldsymbol{S}$ is nonsingular. 


\section{A.2 Proof of Proposition 3.1}

One can compute, following Magnus and Neudecker [30], that

$$
d \boldsymbol{Q}=2\left(\boldsymbol{I}_{p}-\boldsymbol{X}_{\varphi}\right)^{-1} d \boldsymbol{X}_{\boldsymbol{\varphi}}\left(\boldsymbol{I}_{p}-\boldsymbol{X}_{\varphi}\right)^{-1} \boldsymbol{I}_{p \times k},
$$

so that

$$
d \operatorname{vec} \boldsymbol{Q}=2\left[\boldsymbol{I}_{p \times k}^{T}\left(\boldsymbol{I}_{p}-\boldsymbol{X}_{\boldsymbol{\varphi}}\right)^{-T} \otimes\left(\boldsymbol{I}_{p}-\boldsymbol{X}_{\boldsymbol{\varphi}}^{-1}\right)\right] d \operatorname{vec} \boldsymbol{X}_{\boldsymbol{\varphi}}
$$

By Lemma 3.0.1, we have that vec $X_{\varphi}=\Gamma_{\mathcal{V}} \boldsymbol{\varphi}$. Thus,

$$
d \operatorname{vec} \boldsymbol{Q}=2\left[\boldsymbol{I}_{p \times k}^{T}\left(\boldsymbol{I}_{p}-\boldsymbol{X}_{\boldsymbol{\varphi}}\right)^{-T} \otimes\left(\boldsymbol{I}_{p}-\boldsymbol{X}_{\boldsymbol{\varphi}}\right)^{-1}\right] \boldsymbol{\Gamma}_{\mathcal{V}} d \boldsymbol{\varphi}
$$

Using the first identification table of Magnus and Neudecker [30], we identify the derivative matrix

$$
\operatorname{DCay}_{\mathcal{V}}(\boldsymbol{\varphi})=2\left[\boldsymbol{I}_{p \times k}^{T}\left(\boldsymbol{I}_{p}-\boldsymbol{X}_{\boldsymbol{\varphi}}\right)^{-T} \otimes\left(\boldsymbol{I}_{p}-\boldsymbol{X}_{\boldsymbol{\varphi}}\right)^{-1}\right] \boldsymbol{\Gamma}_{\mathcal{V}}
$$

\section{A.3 Proof of Proposition 3.2}

We first show that $l$ is injective on $\mathcal{V}^{+}(k, p)$. Let $\boldsymbol{Q}=\left[\boldsymbol{Q}_{1}^{T} \boldsymbol{Q}_{2}^{T}\right]^{T}, \boldsymbol{Q}^{\prime}=\left[\boldsymbol{Q}_{1}^{\prime T} \boldsymbol{Q}_{2}^{\prime T}\right]^{T} \in$ $\mathcal{V}^{+}(k, p)$ and suppose that $l(\boldsymbol{Q})=l\left(\boldsymbol{Q}^{\prime}\right)$, i.e. the columns of $\boldsymbol{Q}$ and $\boldsymbol{Q}^{\prime}$ span the same subspace. There must exist $\boldsymbol{R} \in \mathcal{O}(k)$ such that $\boldsymbol{Q}=\boldsymbol{Q}^{\prime} \boldsymbol{R}$ so that $\boldsymbol{Q}_{1}=\boldsymbol{Q}_{1}^{\prime} \boldsymbol{R}$. Because the matrix $\boldsymbol{Q}_{1}$ is nonsingular, its left polar decomposition into the product of a symmetric positive definite matrix and an orthogonal matrix is unique (see, for example, Proposition 5.5 of Eaton [16]). We conclude that $\boldsymbol{R}=\boldsymbol{I}_{k}$ and $\boldsymbol{Q}_{1}=\boldsymbol{Q}_{1}^{\prime}$. Thus, $l$ is injective on $\mathcal{V}^{+}(k, p)$.

Next, we prove that the image of $\mathcal{V}^{+}(k, p)$ under $l$ has measure one with respect to the uniform probability measure $P_{\mathcal{G}(k, p)}$ on $\mathcal{G}(k, p)$. Define $\mathcal{V}^{\mathrm{N}}(k, p)$ as the set

$$
\mathcal{V}^{\mathrm{N}}(k, p)=\left\{\boldsymbol{Q}=\left[\begin{array}{ll}
\boldsymbol{Q}_{1}^{T} & \boldsymbol{Q}_{2}^{T}
\end{array}\right]^{T} \in \mathcal{V}(k, p): \boldsymbol{Q}_{1} \text { is nonsingular }\right\} .
$$

The set $\mathcal{V}^{\mathrm{N}}(k, p)$ has measure one with respect to $P_{\mathcal{V}(k, p)}$ and the following lemma holds:

Lemma A.0.1. The images of $\mathcal{V}^{+}(k, p)$ and $\mathcal{V}^{N}(k, p)$ under $l$ are equal. 
Proof. The direction $l\left[\mathcal{V}^{+}(k, p)\right] \subseteq l\left[\mathcal{V}^{\mathrm{N}}(k, p)\right]$ is immediate. Now, let $S \in l\left[\mathcal{V}^{\mathrm{N}}(k, p)\right]$. There must exist $\boldsymbol{Q}=\left[\boldsymbol{Q}_{1}^{T} \boldsymbol{Q}_{2}^{T}\right]^{T} \in \mathcal{V}^{\mathrm{N}}(k, p)$ having $S$ as its column space. Let $\boldsymbol{Q}_{1}=\boldsymbol{U} \boldsymbol{D} \boldsymbol{V}^{T}$ be the singular value decomposition of $\boldsymbol{Q}_{1}$ and set $\boldsymbol{Q}^{\prime}=\boldsymbol{Q} \boldsymbol{V} \boldsymbol{U}^{T}$. Then $l\left(\boldsymbol{Q}^{\prime}\right)=S$ because $\boldsymbol{V} \boldsymbol{U}^{T} \in \mathcal{O}(k)$ and $\boldsymbol{Q}^{\prime} \in \mathcal{V}^{+}(k, p)$ because its square top block $\boldsymbol{U} \boldsymbol{D} \boldsymbol{U}^{T}$ is symmetric positive definite. Thus $S \in l\left[\mathcal{V}^{+}(k, p)\right]$ and we conclude that $l\left[\mathcal{V}^{\mathrm{N}}(k, p)\right] \subseteq l\left[\mathcal{V}^{+}(k, p)\right]$.

Recall that the measure $P_{\mathcal{G}(k, p)}$ is the pushforward of $P_{\mathcal{V}(k, p)}$ by $l$, i.e. for a subset $A \subset \mathcal{G}(k, p)$ we have $P_{\mathcal{G}(k, p)}(A)=P_{\mathcal{V}(k, p)}\left[l^{-1}(A)\right]$. Thus,

$$
\begin{aligned}
P_{\mathcal{G}(k, p)}\left\{l\left[\mathcal{V}^{+}(k, p)\right]\right\} & =P_{\mathcal{G}(k, p)}\left\{l\left[\mathcal{V}^{\mathrm{N}}(k, p)\right]\right\} \\
& =P_{\mathcal{V}(k, p)}\left\{\mathcal{V}^{\mathrm{N}}(k, p)\right\} \\
& =1 .
\end{aligned}
$$

\section{A.4 Proof of Proposition 3.3}

We begin with $\boldsymbol{Q}=\left[\boldsymbol{Q}_{1}^{T} \boldsymbol{Q}_{2}^{T}\right] \in \mathcal{V}^{+}(k, p)$ and we want to verify that the matrix $\boldsymbol{A}$ obtained by equations $4-5$ satisfies $\operatorname{eval}_{i}\left(\boldsymbol{A}^{T} \boldsymbol{A}\right) \in[0,1)$ for $1 \leq i \leq k$. Let $\boldsymbol{Q}_{1}=\boldsymbol{V} \operatorname{diag}\left(\lambda_{1}, \ldots, \lambda_{k}\right) \boldsymbol{V}^{T}$ be the eigendecomposition of $\boldsymbol{Q}_{1}$. That each eigenvalue is positive follows from the condition $\boldsymbol{Q}_{1} \succ \mathbf{0}$. We know that each eigenvalue is less than or equal to one because

$$
\begin{aligned}
\mathbf{0}_{k} & \preceq \boldsymbol{Q}_{2}^{T} \boldsymbol{Q}_{2} \\
& =\boldsymbol{I}_{k}-\boldsymbol{Q}_{1}^{T} \boldsymbol{Q}_{1} \\
& =\boldsymbol{V} \operatorname{diag}\left(1-\lambda_{1}^{2}, \ldots, 1-\lambda_{k}^{2}\right) \boldsymbol{V}^{T} .
\end{aligned}
$$

Therefore, $\lambda_{i} \in(0,1]$ for each $i$. As in equations 4-5, set

$$
\begin{aligned}
\boldsymbol{F} & =\left(\boldsymbol{I}_{k}-\boldsymbol{Q}_{1}\right)\left(\boldsymbol{I}_{k}+\boldsymbol{Q}_{1}\right)^{-1} \\
\boldsymbol{A} & =\frac{1}{2} \boldsymbol{Q}_{2}\left(\boldsymbol{I}_{k}+\boldsymbol{F}\right) .
\end{aligned}
$$

We can write $\boldsymbol{A}^{T} \boldsymbol{A}$ as

$$
\begin{aligned}
\boldsymbol{A}^{T} \boldsymbol{A} & =\frac{1}{4}\left(\boldsymbol{I}_{k}+\boldsymbol{F}\right)^{T} \boldsymbol{Q}_{2}^{T} \boldsymbol{Q}_{2}\left(\boldsymbol{I}_{k}+\boldsymbol{F}\right) \\
& =\frac{1}{4}\left(\boldsymbol{I}_{k}+\boldsymbol{F}\right)^{T}\left(\boldsymbol{I}_{k}-\boldsymbol{Q}_{1}^{T} \boldsymbol{Q}_{1}\right)\left(\boldsymbol{I}_{k}+\boldsymbol{F}\right)
\end{aligned}
$$


from which it follows that

$$
\operatorname{eval}_{i}\left(\boldsymbol{A}^{T} \boldsymbol{A}\right)=\frac{1}{4}\left(1-\lambda_{i}^{2}\right)\left(1+\frac{1-\lambda_{i}}{1+\lambda_{i}}\right)^{2}
$$

for each $i$. Since the eigenvalues of $\boldsymbol{Q}_{1}$ lie in the interval $(0,1]$, the eigenvalues $\boldsymbol{A}^{T} \boldsymbol{A}$ lie in the interval $[0,1)$.

Now we start with $\boldsymbol{A}$ such that $\delta_{i}=\operatorname{eval}_{i}\left(\boldsymbol{A}^{T} \boldsymbol{A}\right) \in[0,1)$ for each $i$ and we want to check that $\boldsymbol{Q}_{1}=\left(\boldsymbol{I}_{k}-\boldsymbol{A}^{T} \boldsymbol{A}\right)\left(\boldsymbol{I}_{k}+\boldsymbol{A}^{T} \boldsymbol{A}\right)^{-1} \succ \mathbf{0}$. Let $\boldsymbol{A}^{T} \boldsymbol{A}=\boldsymbol{W} \operatorname{diag}\left(\delta_{1}, \ldots, \delta_{k}\right) \boldsymbol{W}^{T}$ be the eigendecomposition of $\boldsymbol{A}^{T} \boldsymbol{A}$. Then

$$
\boldsymbol{Q}_{1}=\boldsymbol{W} \operatorname{diag}\left(\frac{1-\delta_{1}}{1+\delta_{1}}, \ldots, \frac{1-\delta_{k}}{1+\delta_{k}}\right) \boldsymbol{W}^{T} \succ \mathbf{0} .
$$

\section{A.5 Proof of Proposition 3.4}

The proof is nearly identical to that of Proposition 3.1. We simply replace $\boldsymbol{\varphi}$ with $\boldsymbol{\psi}$ and $\boldsymbol{\Gamma}_{\mathcal{V}}$ with $\boldsymbol{\Gamma}_{\mathcal{G}}$

\section{A.6 Proof of Proposition 6.1 part (i)}

Denote the square top block of $\boldsymbol{Q}_{p}$ by $\boldsymbol{Q}_{p, 1}$ and the bottom block by $\boldsymbol{Q}_{p, 2}$. Define matrices

$$
\begin{aligned}
\boldsymbol{F}_{p} & =\left(\boldsymbol{I}_{k_{p}}-\boldsymbol{Q}_{p, 1}\right)\left(\boldsymbol{I}_{k_{p}}+\boldsymbol{Q}_{p, 1}\right)^{-1} \\
\boldsymbol{B}_{p} & =\frac{1}{2}\left(\boldsymbol{F}_{p}^{T}-\boldsymbol{F}_{p}\right) \\
\boldsymbol{A}_{p} & =\frac{1}{2} \boldsymbol{Q}_{p, 2}\left(\boldsymbol{I}_{k_{p}}+\boldsymbol{F}_{p}\right)
\end{aligned}
$$

as in equations 1-3. Let $\boldsymbol{b}_{p}$ be the vector of independent entries of $\boldsymbol{B}_{p}$ obtained by eliminating diagonal and supradiagonal elements from vec $\boldsymbol{B}_{p}$. When the Frobenius norm $\left\|\boldsymbol{Q}_{p, 1}\right\|_{F}$ is less than one, the matrices admit series representations:

$$
\begin{aligned}
\boldsymbol{F}_{p} & =\boldsymbol{I}_{k_{p}}-2 \boldsymbol{Q}_{p, 1}+2 \boldsymbol{Q}_{p, 1}^{2}-2 \boldsymbol{Q}_{p, 1}^{3}+\ldots \\
\boldsymbol{B}_{p} & =\left(\boldsymbol{Q}_{p, 1}-\boldsymbol{Q}_{p, 1}^{T}\right)-\left(\boldsymbol{Q}_{p, 1}^{2}-\boldsymbol{Q}_{p, 1}^{2 T}\right)+\left(\boldsymbol{Q}_{p, 1}^{3}-\boldsymbol{Q}_{p, 1}^{3 T}\right)-\ldots \\
\boldsymbol{A}_{p} & =\boldsymbol{Q}_{p, 2}-\boldsymbol{Q}_{p, 2} \boldsymbol{Q}_{p, 1}+\boldsymbol{Q}_{p, 2} \boldsymbol{Q}_{p, 1}^{2}-\boldsymbol{Q}_{2} \boldsymbol{Q}_{p, 1}^{3}+\ldots
\end{aligned}
$$


It follows that

$$
\begin{aligned}
& \widetilde{\boldsymbol{B}}_{p}\left(\boldsymbol{Q}_{p}\right)-\boldsymbol{B}_{p}=\sum_{i=2}^{\infty}(-1)^{i}\left(\boldsymbol{Q}_{p, 1}^{i}-\boldsymbol{Q}_{p, 1}^{i T}\right) \\
& \widetilde{\boldsymbol{A}}_{p}\left(\boldsymbol{Q}_{p}\right)-\boldsymbol{A}_{p}=\sum_{i=1}^{\infty}(-1)^{i-1} \boldsymbol{Q}_{p, 2} \boldsymbol{Q}_{p, 1}^{i}
\end{aligned}
$$

when $\left\|\boldsymbol{Q}_{p, 1}\right\|_{F}<1$. See Hubbard and Hubbard [20] for a discussion of matrix geometric series.

Inequalities relating the Frobenius and max norms will prove useful. Let $\boldsymbol{W}_{1}, \boldsymbol{W}_{2}$ be $d_{1} \times d_{2}$ and $d_{2} \times d_{3}$ dimensional matrices, respectively. Then

$$
\begin{gathered}
\left\|\boldsymbol{W}_{1}\right\|_{F} \leq \sqrt{d_{1} d_{2}}\left\|\boldsymbol{W}_{1}\right\|_{\max } \\
\left\|\boldsymbol{W}_{1} \boldsymbol{W}_{2}\right\|_{\max } \leq d_{2}\left\|\boldsymbol{W}_{1}\right\|_{\max }\left\|\boldsymbol{W}_{2}\right\|_{\max }
\end{gathered}
$$

The first inequality implies that the condition $\left\|\boldsymbol{Q}_{p, 1}\right\|_{F}<1$ under which our series representations converge is satisfied when $k_{p}\left\|\boldsymbol{Q}_{p, 1}\right\|_{\max }<1$. The second inequality implies that $\left\|\boldsymbol{Q}_{p, 1}^{i}\right\|_{\max } \leq k_{p}^{i-1}\left\|\boldsymbol{Q}_{p, 1}\right\|_{\max }^{i}$ for each natural number $i$.

We will also need the following somewhat technical lemma:

Lemma A.0.2. The following quantities, which will appear in later inequalities, go to zero in probability as $p$ grows provided that $k_{p}=o\left(\frac{p^{1 / 4}}{\sqrt{\log p}}\right)$ :

$$
\begin{array}{lll}
\text { (i) } \quad\left\|p^{1 / 2} \boldsymbol{Q}_{p}-\boldsymbol{Z}_{p}\right\|_{\max } & \text { (ii) } & k_{p}\left\|\boldsymbol{Q}_{p, 1}\right\|_{\max } \\
\text { (iii) } \quad k_{p}^{2} p^{1 / 2}\left\|\boldsymbol{Q}_{p, 1}\right\|_{\max }^{2} & \text { (iv) } & k_{p} p^{1 / 2}\left\|\boldsymbol{Q}_{p}\right\|_{\max }^{2}
\end{array}
$$

Proof. Suppose $k_{p}=o\left(\frac{p^{1 / 4}}{\sqrt{\log p}}\right)$. This implies that $k_{p}=o\left(\frac{p}{\log p}\right)$ and quantity (i) goes to zero in probability by Theorem 3 of Jiang [23]. The quantities (ii)-(iv) are nonnegative and each is smaller than either $k_{p}^{2} p^{1 / 2}\left\|\boldsymbol{Q}_{p}\right\|_{\text {max }}^{2}$ or its square root. If we can show that either this quantity or its square root goes to zero in probability, we are done. We have the following upper bound for the square root:

$$
\begin{aligned}
k_{p} p^{1 / 4}\left\|\boldsymbol{Q}_{p}\right\|_{\max } & =k_{p} p^{1 / 4}\left\|\boldsymbol{Q}_{p}-p^{-1 / 2} \boldsymbol{Z}_{p}+p^{-1 / 2} \boldsymbol{Z}_{p}\right\|_{\max } \\
& \leq k_{p} p^{1 / 4}\left(\left\|\boldsymbol{Q}_{p}-p^{-1 / 2} \boldsymbol{Z}_{p}\right\|_{\max }+\left\|p^{-1 / 2} \boldsymbol{Z}_{p}\right\|_{\max }\right)
\end{aligned}
$$




$$
=k_{p} p^{-1 / 4}\left\|p^{1 / 2} \boldsymbol{Q}_{p}-\boldsymbol{Z}_{p}\right\|_{\max }+k_{p} p^{-1 / 4}\left\|\boldsymbol{Z}_{p}\right\|_{\max } .
$$

We know that the first summand goes to zero in probability by the condition on $k_{p}$ and the fact that quantity (i) goes to zero in probability. Using a well-known inequality involving the maximum of independent standard normal random variables (see Problem 5.1 in van Handel [47]), we obtain the following bound on the expected value of the second summand:

$$
\begin{aligned}
k_{p} p^{-1 / 4} \mathbb{E}\left[\left\|\boldsymbol{Z}_{p}\right\|_{\max }\right] & \leq k_{p} p^{-1 / 4} \sqrt{2 \log p k_{p}} \\
& \leq k_{p} p^{-1 / 4} \sqrt{2 \log p^{2}} \\
& =2 \frac{k_{p}}{\left(\frac{p^{1 / 4}}{\sqrt{\log p}}\right)} .
\end{aligned}
$$

The condition on $k_{p}$ implies that the expectation of the second summand goes to zero. We conclude that the second summand goes to zero in mean and thus in probability. We have shown that an upper bound for the square root of $k_{p}^{2} p^{1 / 2}\left\|\boldsymbol{Q}_{p}\right\|_{\max }^{2}$ goes to zero in probability, which is sufficient to prove the lemma.

Now set $a_{p}=\left\|\boldsymbol{\Pi}_{p} \widetilde{\boldsymbol{\varphi}}_{p}-\boldsymbol{\Pi}_{p} \boldsymbol{\varphi}_{p}\right\|_{\infty}$, assume that $k_{p}=o\left(\frac{p^{1 / 4}}{\sqrt{\log p}}\right)$, and let $\epsilon>0$ be given. We want to show that $\operatorname{Pr}\left\{a_{p}>\epsilon\right\} \rightarrow 0$ as $p$ gets large. We express this probability as

$$
\begin{aligned}
\operatorname{Pr}\left\{a_{p}>\epsilon\right\}= & \operatorname{Pr}\left\{a_{p}>\epsilon \mid k_{p}\left\|\boldsymbol{Q}_{p, 1}\right\|_{\max }<1\right\} \operatorname{Pr}\left\{k_{p}\left\|\boldsymbol{Q}_{p, 1}\right\|_{\max }<1\right\}+ \\
& \operatorname{Pr}\left\{a_{p}>\epsilon \mid k_{p}\left\|\boldsymbol{Q}_{p, 1}\right\|_{\max } \geq 1\right\} \operatorname{Pr}\left\{k_{p}\left\|\boldsymbol{Q}_{p, 1}\right\|_{\max } \geq 1\right\} \\
\leq & \operatorname{Pr}\left\{a_{p}>\epsilon \mid k_{p}\left\|\boldsymbol{Q}_{p, 1}\right\|_{\max }<1\right\}+\operatorname{Pr}\left\{k_{p}\left\|\boldsymbol{Q}_{p, 1}\right\|_{\max } \geq 1\right\}
\end{aligned}
$$

Lemma A.0.2 implies that $\operatorname{Pr}\left\{k_{p}\left\|\boldsymbol{Q}_{p, 1}\right\|_{\max } \geq 1\right\}$ goes to zero. It follows that $\operatorname{Pr}\left\{a_{p}>\epsilon\right\}$ goes to zero if $\operatorname{Pr}\left\{a_{p}>\epsilon \mid k_{p}\left\|\boldsymbol{Q}_{p, 1}\right\|_{\max }<1\right\}$ does. Therefore, we only need to verify this condition.

For each $p$, we have

$$
\begin{aligned}
a_{p} & =\left\|\boldsymbol{\Pi}_{p} \widetilde{\boldsymbol{\varphi}}_{p}-\boldsymbol{\Pi}_{p} \boldsymbol{\varphi}_{p}\right\|_{\infty} \\
& =\left\|\left[\begin{array}{c}
\sqrt{p / 2} \widetilde{\boldsymbol{b}}_{p}\left(\boldsymbol{Q}_{p}\right) \\
\sqrt{p} \operatorname{vec} \widetilde{\boldsymbol{A}}_{p}\left(\boldsymbol{Q}_{p}\right)
\end{array}\right]-\left[\begin{array}{c}
\sqrt{p / 2} \boldsymbol{b}_{p} \\
\sqrt{p} \operatorname{vec} \boldsymbol{A}_{p}
\end{array}\right]\right\|_{\infty} \\
& =\max \left\{\sqrt{p / 2}\left\|\widetilde{\boldsymbol{B}}_{p}\left(\boldsymbol{Q}_{p}\right)-\boldsymbol{B}_{p}\right\|_{\max }, \sqrt{p}\left\|\widetilde{\boldsymbol{A}}_{p}\left(\boldsymbol{Q}_{p}\right)-\boldsymbol{A}_{p}\right\|_{\max }\right\}
\end{aligned}
$$




$$
\leq \sqrt{p / 2}\left\|\widetilde{\boldsymbol{B}}_{p}\left(\boldsymbol{Q}_{p}\right)-\boldsymbol{B}_{p}\right\|_{\max }+\sqrt{p}\left\|\widetilde{\boldsymbol{A}}_{p}\left(\boldsymbol{Q}_{p}\right)-\boldsymbol{A}_{p}\right\|_{\max } .
$$

When $k_{p}\left\|\boldsymbol{Q}_{p, 1}\right\|_{\max }<1$, we have

$$
\begin{aligned}
\sqrt{p / 2}\left\|\widetilde{\boldsymbol{B}}_{p}\left(\boldsymbol{Q}_{p}\right)-\boldsymbol{B}_{p}\right\|_{\max } & =\sqrt{p / 2}\left\|\sum_{i=2}^{\infty}(-1)^{i}\left(\boldsymbol{Q}_{p, 1}^{i}-\boldsymbol{Q}_{p, 1}^{i T}\right)\right\|_{\max } \\
& \leq \sqrt{p / 2} \sum_{i=2}^{\infty}\left\|\boldsymbol{Q}_{p, 1}^{i}-\boldsymbol{Q}_{p, 1}^{i T}\right\|_{\max } \\
& \leq \sqrt{p / 2} \sum_{i=2}^{\infty} 2\left\|\boldsymbol{Q}_{p, 1}^{i}\right\|_{\max } \\
& =\sqrt{2 p} \sum_{i=2}^{\infty}\left\|\boldsymbol{Q}_{p, 1}^{i}\right\|_{\max } \\
& \leq \sqrt{2 p} \sum_{i=2}^{\infty} k_{p}^{i-1}\left\|\boldsymbol{Q}_{p, 1}\right\|_{\max }^{i} \\
& =\sqrt{2 p} \sum_{n=i}^{\infty}\left(k_{p}^{\frac{i-1}{i}}\left\|\boldsymbol{Q}_{p, 1}\right\|_{\max }\right)^{i} \\
& \leq \sqrt{2 p} \sum_{i=2}^{\infty}\left(k_{p}\left\|\boldsymbol{Q}_{p, 1}\right\|_{\max }\right)^{i} \\
& =\sqrt{2 p}\left(\frac{1}{1-k_{p}\left\|\boldsymbol{Q}_{p, 1}\right\|_{\max }}-k_{p}\left\|\boldsymbol{Q}_{p, 1}\right\|_{\max }-1\right) \\
& =\sqrt{2} \frac{k_{p}^{2} \sqrt{p}\left\|\boldsymbol{Q}_{p, 1}\right\|_{\max }^{2}}{1-k_{p}\left\|\boldsymbol{Q}_{p, 1}\right\|_{\max }} \\
& \\
& \\
&
\end{aligned}
$$

and

$$
\begin{aligned}
\sqrt{p}\left\|\widetilde{\boldsymbol{A}}_{p}\left(\boldsymbol{Q}_{p}\right)-\boldsymbol{A}_{p}\right\|_{\max } & =\sqrt{p}\left\|\sum_{i=1}^{\infty}(-1)^{i-1} \boldsymbol{Q}_{p, 2} \boldsymbol{Q}_{p, 1}^{i}\right\|_{\max } \\
& \leq \sqrt{p} \sum_{n=1}^{\infty}\left\|\boldsymbol{Q}_{p, 2} \boldsymbol{Q}_{p, 1}^{n}\right\|_{\max } \\
& \leq \sqrt{p} \sum_{n=1}^{\infty} k_{p}\left\|\boldsymbol{Q}_{p, 2}\right\|_{\max }\left\|\boldsymbol{Q}_{p, 1}^{n}\right\|_{\max } \\
& \leq \sqrt{p}\left\|\boldsymbol{Q}_{p, 2}\right\|_{\max } \sum_{n=1}^{\infty}\left(k_{p}\left\|\boldsymbol{Q}_{p, 1}\right\|_{\max }\right)^{n}
\end{aligned}
$$




$$
\begin{aligned}
& =\sqrt{p}\left\|\boldsymbol{Q}_{p, 2}\right\|_{\max }\left(\frac{1}{1-k_{p}\left\|\boldsymbol{Q}_{p, 1}\right\|_{\max }}-1\right) \\
& =\sqrt{p}\left\|\boldsymbol{Q}_{p, 2}\right\|_{\max }\left(\frac{k_{p}\left\|\boldsymbol{Q}_{p, 1}\right\|_{\max }}{1-k_{p}\left\|\boldsymbol{Q}_{p, 1}\right\|_{\max }}\right) \\
& \leq \frac{k_{p} \sqrt{p} \max \left\{\left\|\boldsymbol{Q}_{p, 1}\right\|_{\max },\left\|\boldsymbol{Q}_{p, 2}\right\|_{\max }\right\}^{2}}{1-k_{p}\left\|\boldsymbol{Q}_{p, 1}\right\|_{\max }} \\
& =\frac{k_{p} \sqrt{p}\left\|\boldsymbol{Q}_{p}\right\|_{\max }^{2}}{1-k_{p}\left\|\boldsymbol{Q}_{p, 1}\right\|_{\max }} .
\end{aligned}
$$

Thus, the upper bound

$$
\begin{aligned}
a_{p} & \leq \sqrt{p / 2}\left\|\widetilde{\boldsymbol{B}}_{p}\left(\boldsymbol{Q}_{p}\right)-\boldsymbol{B}_{p}\right\|_{\max }+\sqrt{p}\left\|\widetilde{\boldsymbol{A}}_{p}\left(\boldsymbol{Q}_{p}\right)-\boldsymbol{A}_{p}\right\|_{\max } \\
& \leq \sqrt{2} \frac{k_{p}^{2} \sqrt{p}\left\|\boldsymbol{Q}_{p, 1}\right\|_{\max }^{2}}{1-k_{p}\left\|\boldsymbol{Q}_{p, 1}\right\|_{\max }}+\frac{k_{p} \sqrt{p}\left\|\boldsymbol{Q}_{p}\right\|_{\max }^{2}}{1-k_{p}\left\|\boldsymbol{Q}_{p, 1}\right\|_{\max }}:=u_{p}
\end{aligned}
$$

is valid when $k_{p}\left\|\boldsymbol{Q}_{p, 1}\right\|_{\max }<1$. Then

$$
\begin{aligned}
\operatorname{Pr}\left\{a_{p}>\epsilon \mid k_{p}\left\|\boldsymbol{Q}_{p, 1}\right\|_{\max }<1\right\} & \leq \operatorname{Pr}\left\{u_{p}>\epsilon \mid k_{p}\left\|\boldsymbol{Q}_{p, 1}\right\|_{\max }<1\right\} \\
& =\frac{\operatorname{Pr}\left\{u_{p}>\epsilon \cap k_{p}\left\|\boldsymbol{Q}_{p, 1}\right\|_{\max }<1\right\}}{\operatorname{Pr}\left\{k_{p}\left\|\boldsymbol{Q}_{p, 1}\right\|_{\max }<1\right\}} \\
& \leq \frac{\operatorname{Pr}\left\{u_{p}>\epsilon\right\}}{\operatorname{Pr}\left\{k_{p}\left\|\boldsymbol{Q}_{p, 1}\right\|_{\max }<1\right\}} .
\end{aligned}
$$

Because $\operatorname{Pr}\left\{k_{p}\left\|\boldsymbol{Q}_{p, 1}\right\|_{\max }<1\right\} \rightarrow 1$, we only need to show $\operatorname{Pr}\left\{u_{p}>\epsilon\right\} \rightarrow 0$ as $p$ grows. Since $\epsilon$ is arbitrary, this is equivalent to showing that $u_{p}$ goes to zero in probability, which follows from the continuous mapping theorem and Lemma A.0.2. We have shown that $\operatorname{Pr}\left\{a_{p}>\epsilon \mid k_{p}\left\|\boldsymbol{Q}_{p, 1}\right\|_{\max }<1\right\}$ goes to zero as $p$ gets large, which is sufficient to prove part (i) of the proposition.

\section{A.7 Proof of Proposition 6.1 part (ii)}

Assume that $k_{p}=o\left(\frac{p}{\log p}\right)$. For each $p$, we have:

$$
\begin{aligned}
\left\|\boldsymbol{\Pi}_{p} \widetilde{\boldsymbol{\varphi}}_{p}-\boldsymbol{z}_{p}\right\|_{\infty} & =\left\|\boldsymbol{\Pi}_{p} \widetilde{C}_{p}^{-1}\left(\boldsymbol{Q}_{p}\right)-\boldsymbol{\Pi}_{p} \widetilde{C}_{p}^{-1}\left(p^{-1 / 2} \boldsymbol{Z}_{p}\right)\right\|_{\infty} \\
& =\left\|\left[\begin{array}{c}
\sqrt{p / 2} \widetilde{b}_{p}\left(\boldsymbol{Q}_{p}\right) \\
\sqrt{p} \operatorname{vec} \widetilde{A}_{p}\left(\boldsymbol{Q}_{p}\right)
\end{array}\right]-\left[\begin{array}{c}
\sqrt{p / 2} \widetilde{b}_{p}\left(p^{-1 / 2} \boldsymbol{Z}_{p}\right) \\
\sqrt{p} \operatorname{vec} \widetilde{A}_{p}\left(p^{-1 / 2} \boldsymbol{Z}_{p}\right)
\end{array}\right]\right\|_{\infty} \\
& =\max \left\{\sqrt{p / 2}\left\|\widetilde{B}_{p}\left(\boldsymbol{Q}_{p}\right)-\widetilde{B}_{p}\left(p^{-1 / 2} \boldsymbol{Z}_{p}\right)\right\|_{\max },\right.
\end{aligned}
$$




$$
\begin{aligned}
& \left.\sqrt{p}\left\|\widetilde{A}_{p}\left(\boldsymbol{Q}_{p}\right)-\widetilde{A}_{p}\left(p^{-1 / 2} \boldsymbol{Z}_{p}\right)\right\|_{\max }\right\} \\
\leq & \sqrt{p / 2}\left\|\widetilde{B}_{p}\left(\boldsymbol{Q}_{p}\right)-\widetilde{B}_{p}\left(p^{-1 / 2} \boldsymbol{Z}_{p}\right)\right\|_{\max }+ \\
& \sqrt{p}\left\|\widetilde{A}_{p}\left(\boldsymbol{Q}_{p}\right)-\widetilde{A}_{p}\left(p^{-1 / 2} \boldsymbol{Z}_{p}\right)\right\|_{\max } \\
= & \sqrt{p / 2}\left\|\boldsymbol{Q}_{p, 1}-p^{-1 / 2} \boldsymbol{Z}_{p, 1}+p^{-1 / 2} \boldsymbol{Z}_{p, 1}^{T}-\boldsymbol{Q}_{p, 1}^{T}\right\|_{\max }+ \\
& \sqrt{p}\left\|\boldsymbol{Q}_{p, 2}-p^{-1 / 2} \boldsymbol{Z}_{p, 2}\right\|_{\max } \\
\leq & \sqrt{p / 2}\left(\left\|\boldsymbol{Q}_{p, 1}-p^{-1 / 2} \boldsymbol{Z}_{p, 1}\right\|+\left\|p^{-1 / 2} \boldsymbol{Z}_{p, 1}^{T}-\boldsymbol{Q}_{p, 1}^{T}\right\|_{\max }\right)+ \\
& \sqrt{p}\left\|\boldsymbol{Q}_{p, 2}-p^{-1 / 2} \boldsymbol{Z}_{p, 2}\right\|_{\max } \\
= & 2 \sqrt{p / 2}\left\|\boldsymbol{Q}_{p, 1}-p^{-1 / 2} \boldsymbol{Z}_{p, 1}\right\|_{\max }+\sqrt{p}\left\|\boldsymbol{Q}_{p, 2}-p^{-1 / 2} \boldsymbol{Z}_{p, 2}\right\|_{\max } \\
= & \sqrt{2}\left\|\sqrt{p} \boldsymbol{Q}_{p, 1}-\boldsymbol{Z}_{p, 1}\right\|_{\max }+\left\|\sqrt{p} \boldsymbol{Q}_{p, 2}-\boldsymbol{Z}_{p, 2}\right\|_{\max } \\
\leq & \sqrt{2}\left\|\sqrt{p} \boldsymbol{Q}_{p}-\boldsymbol{Z}_{p}\right\|_{\max }+\left\|\sqrt{p} \boldsymbol{Q}_{p}-\boldsymbol{Z}_{p}\right\|_{\max } \\
= & (\sqrt{2}+1)\left\|\sqrt{p} \boldsymbol{Q}_{p}-\boldsymbol{Z}_{p}\right\|_{\max } .
\end{aligned}
$$

Theorem 3 of Jiang [23] implies that this upper bound goes to zero in probability as $p$ grows. Therefore, the quantity $\left\|\boldsymbol{\Pi}_{p} \widetilde{\boldsymbol{\varphi}}_{p}-\boldsymbol{z}_{p}\right\|_{\infty}$ does as well.

\section{B Special matrices}

In constructing the linear transformations given in Lemmas 3.0.1 and 3.0.2, we rely upon two special matrices: the commutation matrix $\boldsymbol{K}_{m, n}$ and the matrix $\widetilde{\boldsymbol{D}}_{n}$. An early reference related to the matrix $\boldsymbol{K}_{m, n}$ is Magnus and Neudecker [29], while the matrix $\widetilde{\boldsymbol{D}}_{n}$ was introduced in Neudecker [37]. Our presentation follows that of Magnus [28].

\section{B.1 The commutation matrix $\boldsymbol{K}_{m, n}$}

Let $\boldsymbol{A}$ be an $m \times n$ matrix and $B$ be a $p \times q$ matrix. The commutation matrix $\boldsymbol{K}_{m, n}$ is the unique $m n \times m n$ permutation matrix with the property that $\boldsymbol{K}_{m, n} \operatorname{vec} A=$ $\operatorname{vec} A^{T}$. The critical property of the commutation matrix is that it allows us to exchange the order of the matrices in a Kronecker product. Theorem 3.1 of Magnus [28] states that $\boldsymbol{K}_{p, m}(\boldsymbol{A} \otimes \boldsymbol{B})=(\boldsymbol{B} \otimes \boldsymbol{A}) \boldsymbol{K}_{q, n}$. Section 3.3 of Magnus [28] gives an explicit expression for the commutation matrix $\boldsymbol{K}_{m, n}$. Let $\boldsymbol{H}_{i, j}$ be the $m \times n$ matrix having a 1 in the $i, j$ th position and zeros everywhere else. Then Theorem 3.2 of 
Magnus [28] tells us that

$$
\boldsymbol{K}_{m, n}=\sum_{i=1}^{m} \sum_{j=1}^{n}\left(\boldsymbol{H}_{i, j} \otimes \boldsymbol{H}_{i, j}^{T}\right)
$$

\section{B.2 The matrix $\widetilde{\boldsymbol{D}}_{n}$}

The matrix $\widetilde{\boldsymbol{D}}_{n}$ proves useful when differentiating expressions involving skew-symmetric matrices. Let $\boldsymbol{A}$ be an $n \times n$ matrix and let $\tilde{v}(A)$ be the $n(n-1) / 2$-dimensional vector obtained by eliminating diagonal and supradiagonal elements from the vectorization vec $\boldsymbol{A}$. Definition 6.1 of Magnus [28] defines $\widetilde{\boldsymbol{D}}_{n}$ as the unique $n^{2} \times n(n-1) / 2$ matrix with the property $\widetilde{\boldsymbol{D}}_{n} \tilde{v}(A)=\operatorname{vec} \boldsymbol{A}$ for every skew-symmetric matrix $\boldsymbol{A}$. Theorem 6.1 of Magnus [28] gives an explicit expression for $\widetilde{\boldsymbol{D}}_{n}$. Let $\boldsymbol{E}_{i, j}$ be the $n \times n$ matrix with 1 in the $i, j$ th position and zeros everywhere else and set $\tilde{\boldsymbol{T}}_{i, j}=\boldsymbol{E}_{i, j}-\boldsymbol{E}_{j, i}$. Also, let $\tilde{u}_{i, j}$ be the $n(n-1) / 2$-dimensional vector having 1 in its $(j-1) n+i-j(j+1) / 2$ place and zeros everywhere else. Then Theorem 6.1 tells us that

$$
\widetilde{\boldsymbol{D}}_{n}=\sum_{i>j}\left(\operatorname{vec} \tilde{\boldsymbol{T}}_{i, j}\right) \tilde{u}_{i, j}^{T}
$$

\section{Evaluating the Jacobian terms}

Taking a naive approach to evaluating the Jacobian term $J_{d_{\mathcal{V}}} C(\varphi)$ becomes prohibitively expensive for even small dimensions. Recall that

$$
\begin{aligned}
J_{d_{\mathcal{V}}} C(\boldsymbol{\varphi}) & =\left|D C(\boldsymbol{\varphi})^{T} D C(\boldsymbol{\varphi})\right|^{1 / 2} \\
& =\left|2^{2} \boldsymbol{\Gamma}_{\mathcal{V}}^{T}\left(\boldsymbol{G}_{\mathcal{V}} \otimes \boldsymbol{H}_{\mathcal{V}}\right) \boldsymbol{\Gamma}_{\mathcal{V}}\right|^{1 / 2}
\end{aligned}
$$

where

$$
\begin{aligned}
& \boldsymbol{G}_{\mathcal{V}}=\left(\boldsymbol{I}_{p}-\boldsymbol{X}_{\boldsymbol{\varphi}}\right)^{-1} \boldsymbol{I}_{p \times k} \boldsymbol{I}_{p \times k}^{T}\left(\boldsymbol{I}_{p}-\boldsymbol{X}_{\boldsymbol{\varphi}}\right)^{-T} \\
& \boldsymbol{H}_{\mathcal{V}}=\left(\boldsymbol{I}_{p}-\boldsymbol{X}_{\boldsymbol{\varphi}}\right)^{-T}\left(\boldsymbol{I}_{p}-\boldsymbol{X}_{\boldsymbol{\varphi}}\right)^{-1}
\end{aligned}
$$

The Kronecker product $\boldsymbol{G}_{\mathcal{V}} \otimes \boldsymbol{H}_{\mathcal{V}}$ has dimension $p^{2} \times p^{2}$. Evaluating this Kronecker product and computing its matrix product with $\boldsymbol{\Gamma}_{\mathcal{V}}$ is extremely costly for large $p$. In this section, we describe a more efficient approach which takes advantage of the block stucture of the matrices involved. (The Jacobian term $J_{d_{\mathcal{G}}} C(\boldsymbol{\psi})$ can be 
evaluated analogously.)

Let $\boldsymbol{C}_{\mathcal{V}}=\left(\boldsymbol{I}-\boldsymbol{X}_{\boldsymbol{\varphi}}\right)^{-1}$. Then

$$
C_{\mathcal{V}}=\left[\begin{array}{ll}
C_{11} & C_{12} \\
C_{21} & C_{22}
\end{array}\right]
$$

where

$$
\begin{array}{ll}
\boldsymbol{C}_{11}=\left(\boldsymbol{I}_{k}-\boldsymbol{B}+\boldsymbol{A}^{T} \boldsymbol{A}\right)^{-1} & \boldsymbol{C}_{12}=-\boldsymbol{C}_{11} \boldsymbol{A}^{T} \\
\boldsymbol{C}_{21}=\boldsymbol{A} \boldsymbol{C}_{11} & \boldsymbol{C}_{22}=\boldsymbol{I}_{p-k}-\boldsymbol{A} \boldsymbol{C}_{11} \boldsymbol{A}^{T} .
\end{array}
$$

The blocks of the matrices

$$
\boldsymbol{G}_{\mathcal{V}}=\left[\begin{array}{ll}
\boldsymbol{G}_{11} & \boldsymbol{G}_{12} \\
\boldsymbol{G}_{21} & \boldsymbol{G}_{22}
\end{array}\right] \quad \boldsymbol{H}_{\mathcal{V}}=\left[\begin{array}{ll}
\boldsymbol{H}_{11} & \boldsymbol{H}_{12} \\
\boldsymbol{H}_{21} & \boldsymbol{H}_{22}
\end{array}\right]
$$

can be written in terms of the blocks of $\boldsymbol{C}_{\mathcal{V}}$ as

$$
\begin{array}{lll}
\boldsymbol{H}_{11}=\boldsymbol{C}_{11}^{T} \boldsymbol{C}_{11}+\boldsymbol{C}_{21}^{T} \boldsymbol{C}_{21} & \boldsymbol{H}_{12}=\boldsymbol{C}_{11}^{T} \boldsymbol{C}_{12}+\boldsymbol{C}_{21}^{T} \boldsymbol{C}_{22} \\
\boldsymbol{H}_{21}=\boldsymbol{C}_{12}^{T} \boldsymbol{C}_{11}+\boldsymbol{C}_{22}^{T} \boldsymbol{C}_{21} & \boldsymbol{H}_{22}=\boldsymbol{C}_{12}^{T} \boldsymbol{C}_{12}+\boldsymbol{C}_{22}^{T} \boldsymbol{C}_{22}
\end{array}
$$

and

$$
\begin{aligned}
\boldsymbol{G}_{11} & =\boldsymbol{C}_{11} \boldsymbol{C}_{11}^{T} & \boldsymbol{G}_{12} & =\boldsymbol{C}_{11} \boldsymbol{C}_{21}^{T} \\
\boldsymbol{G}_{21} & =\boldsymbol{C}_{21} \boldsymbol{C}_{11}^{T} & \boldsymbol{G}_{22} & =\boldsymbol{C}_{21} \boldsymbol{C}_{21}^{T} .
\end{aligned}
$$

We can express the matrix $D C(\boldsymbol{\varphi})^{T} D C(\boldsymbol{\varphi})$ in blocks as

$$
\begin{aligned}
D C(\boldsymbol{\varphi})^{T} D C(\boldsymbol{\varphi}) & =2^{2} \boldsymbol{\Gamma}_{\mathcal{V}}^{T}\left(\boldsymbol{G}_{\mathcal{V}} \otimes \boldsymbol{H}_{\mathcal{V}}\right) \boldsymbol{\Gamma}_{\mathcal{V}} \\
& =2^{2}\left[\begin{array}{ll}
\boldsymbol{\Omega}_{11} & \boldsymbol{\Omega}_{12} \\
\boldsymbol{\Omega}_{21} & \boldsymbol{\Omega}_{22}
\end{array}\right]
\end{aligned}
$$


where

$$
\begin{aligned}
& \boldsymbol{\Omega}_{11}=\widetilde{\boldsymbol{D}}_{k}^{T}\left(\boldsymbol{G}_{11} \otimes \boldsymbol{H}_{11}\right) \widetilde{\boldsymbol{D}}_{k} \\
& \boldsymbol{\Omega}_{12}=\widetilde{\boldsymbol{D}}_{k}^{T}\left(\boldsymbol{G}_{11} \otimes \boldsymbol{H}_{12}\right)-\widetilde{\boldsymbol{D}}_{k}^{T}\left(\boldsymbol{G}_{12} \otimes \boldsymbol{H}_{11}\right) \boldsymbol{K}_{p-k, k} \\
& \boldsymbol{\Omega}_{21}=\boldsymbol{\Omega}_{12}^{T} \\
& \boldsymbol{\Omega}_{22}=\left(\boldsymbol{G}_{11} \otimes \boldsymbol{H}_{22}+\boldsymbol{H}_{11} \otimes \boldsymbol{G}_{22}\right)-\left(\boldsymbol{G}_{12} \otimes \boldsymbol{H}_{21}+\boldsymbol{H}_{12} \otimes \boldsymbol{G}_{21}\right) \boldsymbol{K}_{p-k, k}
\end{aligned}
$$

Then

$$
\begin{aligned}
J_{d_{\mathcal{V}}} C(\boldsymbol{\varphi}) & =\left|2^{2}\left[\begin{array}{ll}
\boldsymbol{\Omega}_{11} & \boldsymbol{\Omega}_{12} \\
\boldsymbol{\Omega}_{21} & \boldsymbol{\Omega}_{22}
\end{array}\right]\right|^{1 / 2} \\
& =2^{d_{\mathcal{V}}}\left|\boldsymbol{\Omega}_{22}\right|\left|\boldsymbol{\Omega}_{11}-\boldsymbol{\Omega}_{12} \boldsymbol{\Omega}_{22}^{-1} \boldsymbol{\Omega}_{21}\right|
\end{aligned}
$$

The Kronecker products in the formulas for $\boldsymbol{\Omega}_{11}, \boldsymbol{\Omega}_{12}, \boldsymbol{\Omega}_{21}$, and $\boldsymbol{\Omega}_{22}$ are much smaller than $\boldsymbol{G}_{\mathcal{V}} \otimes \boldsymbol{H}_{\mathcal{V}}$, which leads to a significant computational savings compared to the naive approach. 